\title{
Aggregation Behavior of Model Asphaltenes Revealed from Large-Scale Coarse-Grained Molecular Simulations
}

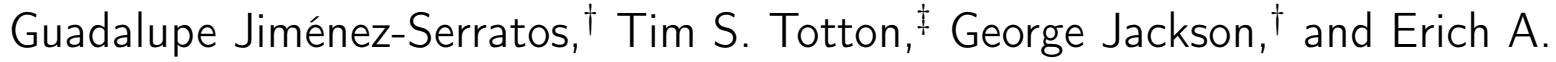 \\ Müller ${ }^{* \dagger}$ \\ $\dagger$ Department of Chemical Engineering, Imperial College London, London SW7 2AZ, U.K. \\ $\ddagger B P$ Exploration Operating Co. Ltd., Sunbury-on-Thames TW16 7LN, U.K. \\ E-mail: eamuller@ic.ac.uk
}

\begin{abstract}
Fully atomistic simulations of models of asphaltenes in simple solvents have allowed the study of trends in aggregation phenomena and the understanding of the role that molecular structure plays therein. However, the detail included at this scale of molecular modeling is at odds with the required spatial and temporal resolution needed to fully understand the asphaltene aggregation. The computational cost required to explore the relevant scales can be reduced by employing coarse-grained (CG) models, which consist of lumping a few atoms into a single segment that is characterised by effective interactions. In this work CG force fields developed via the SAFT- $\gamma$ [Müller, E.A., Jackson, G. (2014) Annu. Rev. Chem. Biomolec. Eng., 5, 405-427] equation of state (EoS) provide a reliable pathway to link the molecular description with macroscopic thermophysical data. A recent modification of the SAFT-VR EoS [Müller, E.A. and Mejía, A. (2017) Langmuir, 33, 11518-11529], that allows parametrizing homonuclear rings, is selected as the starting point to propose CG models for polycyclic aromatic hydrocarbons (PAHs).
\end{abstract}


The new aromatic-core parameters, along with others published for simpler organic molecules, are adopted for the construction of asphaltene models by combining different chemical moieties in a group-contribution fashion. We apply the procedure to two previously reported asphaltene models and perform Molecular Dynamics simulations to validate the coarse-grained representation against benchmark systems of 27 asphaltenes in pure solvent (toluene or heptane) described in a fully atomistic fashion. An excellent match between both levels of description is observed for cluster size, radii of gyration, and relative-shape-anisotropy-factor distributions. We exploit the advantages of the CG representation by simulating systems containing up to 2000 asphaltene molecules in explicit solvent investigating the effect of asphaltene concentration, solvent composition, and temperature on aggregation. Upon employing large systems facilitated by the CG models, we observe stable continuous distributions of molecular aggregates at conditions away from the two-phase precipitation point. As a further example application, a widely accepted interpretation of cluster-size distributions in asphaltenic systems is challenged by performing system-size tests, reversibility proofs and time-dependence analysis. The coarse-graining procedure proposed is seen to be general and predictive, hence, can be applied to other asphaltenic molecular structures.

\section{Introduction}

Asphaltenes are polyaromatic, polar, and heavy molecules present in crude oils ${ }^{1,2}$. The undesired deposition of these molecules in oil industry processes have motivated an extensive research effort to understand the dominant factors affecting asphaltene aggregation with the aim of providing possible solutions to prevent or remediate the problem. Operationally, asphaltenes are defined as the fraction of a crude oil soluble in aromatics, like benzene and toluene, and insoluble in alkanes, like pentane and heptane ${ }^{3-6}$. As a result of this rather broad definition and the widely varying geochemical nature of the precursor oils, it follows that asphaltenes are not unique, well-defined molecules, but rather constitute a very polydisperse 
and complex mixture. In an attempt to simplify their modeling, a wide variety of molecular models have been proposed ${ }^{2,7}$ with structures that can be sorted into two prototypical categories: "continental", in which asphaltenes are represented as polyaromatic cores (of four to ten aromatic rings) with some aliphatic branches, and "archipelago", in which asphaltenes are modeled as small aromatic regions bridged by alkanes. A small amount of heteroatoms, mainly sulphur, nitrogen, and oxygen, is also identified as an important feature of asphaltenic molecules. Supporting evidence of continental structures, archipelago structures, or a mixture of both as the correct representation can be found in the literature ${ }^{2,8-14}$. More controversial, however, is the interpretation and modeling of the aggregation process ${ }^{8,15-23}$. A common interpretation follows the Yen-Mullins model ${ }^{15}$, according to which continental asphaltenes stack in groups of less than 10 molecules to form nanoaggregates of $\sim 2 \mathrm{~nm}$ and upon an increase in the asphaltene concentration, clusters of $\sim 5 \mathrm{~nm}$ formed of about eight of said nanoaggregates, appear. Other models explain aggregation using analogies with micellar systems, which implies a predominant size of the aggregates ("micelles") and an equivalent critical mass concentration. One of the major criticisms of the micellar model is that asphaltenes (or resins, a related smaller molecular weight fraction) lack the molecular features of surfactants (monodispersity, symmetry, and amphiphilic nature) needed to establish deep parallels between both types of systems. A more fundamentally sound treatment ${ }^{8}$ supported that asphaltenes are in solution under favorable conditions (low concentrations, aromatic solvents, high temperatures) and that once those conditions are perturbed, a liquid-liquid phase separation occurs. Sirota ${ }^{8,16}$ suggested that high viscosity of the asphaltenic systems in transition hinders the detection of the phase separation and that the experimental structural data of the glassy state can be misinterpreted if the colloidal assumption is adopted. According to Gray et al. ${ }^{21}$, none of the aggregation models provide a full explanation of different aggregation, deposition, and adsorption phenomena in asphaltenic systems. The authors proposed a supramolecular aggregation model and stated that both paradigms, the micellar and the phase-separation one, intersect when using a solubility parameter. Fogler 
and co-workers ${ }^{17}$ judged inappropriate a sharp distinction in the transition from stable to destabilized asphaltenes, showing the strong-time dependence that exists in the detection of the aggregates in different samples. Acevedo et al. ${ }^{24}$ defined two asphaltene subfractions differentiated by their solubility properties and applied the concept of suppression of phase separation to model nanoaggregate formation. From the above abridged overview it becomes clear that there are many conflicting views and there is the need to explore the aggregation process from a fundamental molecular level. Further literature and discussion on asphaltene structure elucidation, aggregation modeling, and adsorption are presented in the reviews of References 2,7,8,15,19,20,25-27.

Regarding the use of molecular simulations to study asphaltenic systems, all-atom and united-atom (grouping each carbon with the corresponding bonded hydrogens) descriptions have been implemented to study asphaltene systems of different complexity to calculate dimerization energies, research the correlation between functionality and aggregation behavior, study adsorption or interfacial behavior, as well as to obtain a variety of properties, like viscosity, structure factor, nanoaggregate properties, etc. ${ }^{28-42}$. As a consequence of the level of detail of the atomistic representation and the corresponding large computational requirements, the simulated systems usually span only a few nanometers in size and explore events that occur in the order of tens of nanoseconds ${ }^{28,31,37}$, which limits the study of the aggregation phenomenon. To increase size and speed in these molecular simulations, a coarse-grained $(\mathrm{CG})$ description should be adopted ${ }^{43}$. CG, in this context, refers to the representation of a group of atoms by a single segment with effective interactions obtained through a variety of strategies. The so-called "bottom-up" approaches integrate degrees of freedom from lower (more-detailed) descriptions. These treatments involve either quantum mechanical density functional theory $(\mathrm{DFT})^{44}$, computationally expensive iterative inversion methods, where fully-atomistic simulations are used to obtain reference properties that are related to the effective potentials for each $\mathrm{CG}$ freedom degree ${ }^{45,46}$, or development of effective pairwise interactions to match forces on CG sites with those on the reference atom- 
istic system ${ }^{47}$. A more promising approach, based on evaluating the relative entropy, has also been recently proposed ${ }^{48}$. In the "top-down" methodology proposed by Müller and co-workers $^{49-52}$, a molecular-based equation of state (EoS), the statistical associating fluid theory $(\mathrm{SAFT}-\gamma)$, is exploited by linking thermodynamic (i.e. macroscopic) properties of the fluid with the force-field parameters of the underlying CG model. Also worth mentioning are the MARTINI force fields, which combine top-down and bottom-up parametrizations ${ }^{53}$ to develop general CG sites and are widely used in biomolecular and carbohydrate systems ${ }^{54}$. Other combined approaches that are tailored for specific systems can be found in the literature $^{55-58}$. The lack of transferability (the ability to transfer a moiety corresponding to a functional group in one molecule to another molecule containing the same functional group), representability (the ability of the model to represent the experimental system at different thermodynamic states), and robustness (the ability of a model to provide reliable predictions of different properties), as well as the loss of structural detail are the main pitfalls of the CG models ${ }^{49,59-62}$. The specific model application could be behind the choice of one method or the other, and hence, there is a lack of a universally accepted procedure to obtain the effective potential for the "super-atoms".

In particular, the CG parametrization of polycyclic aromatic hydrocarbons (PAHs), which constitute the aromatic regions in asphaltenic structures, connote some further challenges ${ }^{63-67}$. In a contribution which is very relevant to this study, von Lilienfeld and Andrienko $^{44}$ coarse-grain polycyclic aromatic hydrocarbons by fitting effective Lennard-Jones potentials to density-functional theory calculations, showing that the potential parameters of the CG units are specific to the aromatic molecule in the study. Diverse theoretical and simulation studies related to the understanding of PAHs energetics can be found in the literature $^{68-73}$. The specialized discussion of self-organization of the polyaromatic cores into $\pi-\pi$ electron-bonded stacks is beyond the scope of this manuscript but some remarks of its importance in asphaltene dimerization are included in the review presented by Adams ${ }^{19}$.

In recent literature, we find molecular simulation studies of asphaltenic systems described 
at a coarse-grained level with force fields obtained via a variety of approaches. Lee and coworkers ${ }^{74}$ performed extended X-ray absorption fine structure spectra (EXAFS) experiments that were complemented with molecular dynamics (MD) simulations of 400 continental asphaltenes in different solvents to study the features of the aggregates in each case. The CG models were parametrized with MARTINI force fields, the 7-cycle aromatic core was represented with 12 CG segments to maintain a proportion of carbons per CG bead between 3 and 4, and intramolecular restrictions were taken from the MARTINI framework as well, where dihedral angles were considered to maintain planar ring structures. Ferguson and co-workers ${ }^{55}$ coarse-grained three continental asphaltenes and simulated monodisperse systems of up to 270 asphaltenes in explicit CG heptane or toluene to obtain dynamic and structural properties. The clustering was analyzed in detail using different metrics and the averaged cluster size was used to show a hierarchical assembly, following the YenMullins model. Their coarse-grained models were initially represented using the MARTINI force-fields ${ }^{53}$ with the corresponding Coulomb interactions, but the parametrization of the aromatic-core segments was elegantly improved using potential of mean force (PMF) curves from all-atom simulations as a reference. One of the models was further studied in Ref. 75 to understand the effect of temperature, pressure, and solvent composition on the averaged cluster size. The behavior of asphaltenes at oil-water interfaces was studied by Ervik et al. ${ }^{76}$ using multiscale techniques. They performed large-scale molecular dynamics simulations of CG systems to obtain interfacial properties that were used to feed their proposed hybrid level-set/ghost-fluid/immersed-boundary method. The coarse-grained model was built in a group-contribution fashion using CG force-fields developed via the SAFT- $\gamma$ Mie methodology ${ }^{49}$. Two sets of parameters for the aromatic core were tested and it was shown that the interfacial behavior of asphaltenes had a strong dependence on the selection of the core force-fields. Dissipative particle dynamics (DPD), in which small regions of the solvent are represented as a soft bead, have also been implemented to study asphaltenic systems. For example, Zhang et al. ${ }^{77}$ studied the aggregation of asphaltenes with different morphologies 
and an oil-water emulsion modeled with six components at several compositions. To model the aromatic cores in the asphaltene and resin models, the authors proposed a rigid sheet of particles representing fused aromatic rings, arranged to preserve the aromatic-core topology. Other works in the literature that involve molecular simulations of asphaltenes described at a coarse-grained level are presented in Refs. 20,78-83.

In this work, two atomistically detailed asphaltene models, previously presented in the literature, are coarse-grained and the aggregation properties are studied at different thermodynamic conditions via molecular dynamics simulations. The two models represent the different plausible morphologies (continental and archipelago) thought to be relevant. Both models are formed from homonuclear PAH cores decorated with or connected via aliphatic chains. The aliphatic branches are modeled via corresponding-states correlations and the SAFT- $\gamma \operatorname{EoS}^{52,84}$, while a recent modification of the SAFT-VR EoS ${ }^{63}$ is used as the starting point to produce CG parameters for well-defined PAHs, which then serve as the basis to model the aromatic cores. These CG models are simulated using boxes of 27 asphaltenes in explicit solvent matching the size and thermodynamic conditions of the fully-atomistic simulations in Refs. 28,29, which are used as a benchmark. Direct comparison of the structural properties of both representations is shown in the CG model validation section. The CG continental asphaltene is used in simulations of up to 2000 solute molecules to study the effect of varying the asphaltene concentration, solvent composition, and temperature. The aggregation is quantified via cluster-size distributions, and the trends are reported in the Large-scale simulations section. Some selected cases are further analyzed to show the system-size and time-dependence effects on the simulation results. We further discuss the importance of those tests in the interpretation of simulation results in systems that present aggregation. 


\section{Coarse-graining polycyclic aromatic hydrocarbons}

\section{SAFT- $\gamma$ Mie force fields}

The statistical associating fluid theory $(\mathrm{SAFT})^{85-87}$ is a molecular-based equation of state (EoS) based on Wertheim's first order thermodynamic perturbation theory ${ }^{88}$. Molecules under the SAFT lens are envisaged as linearly connected flexible chains of $m$ spherical segments. The dispersion interactions are modeled through a force-field potential and associating sites can be included in the description to represent strong anisotropies in the interactions, typical of hydrogen-bonding fluids. In its general form, the equation is written for the Helmholtz free energy as

$$
A=A^{\text {ideal }}+A^{\text {mono }}+A^{\text {chain }}+A^{\text {assoc }},
$$

where $A^{\text {ideal }}$ is the ideal term, $A^{\text {mono }}$ is the contribution due to the isotropic repulsion and dispersion forces between individual spherical segments in a chain, $A^{\text {chain }}$ is the contribution due to chain formation from segments, and $A^{a s s o c}$ corresponds to association sites represented in the model. The original SAFT approach, as presented by Chapman et al. ${ }^{86,87}$, has been widely adapted to describe complex fluids. Reviews of the theory and its different versions can be read in Refs. 89-92.

In this work, we employ the SAFT- $\gamma$ Mie version ${ }^{50,51}$, in which the non-bonded segmentsegment interactions are modeled via the analytical Mie potential with the attractive exponent fixed to a value of 6 (according to London dispersion forces):

$$
u(r)=\frac{\lambda}{\lambda-6}\left(\frac{\lambda}{6}\right)^{\frac{6}{\lambda-6}} \epsilon\left[\left(\frac{\sigma}{r}\right)^{\lambda}-\left(\frac{\sigma}{r}\right)^{6}\right]
$$

where $r$ is the distance between centers of a pair of segments, $\lambda$ is the repulsive exponent, $\epsilon$ is the depth of the potential-energy well, and $\sigma$ represents the average segment diameter. In addition, a molecular model is defined by the number $m$ of spherical beads which form part of it. 
In principle, the relevant molecular parameters $(m, \epsilon, \sigma, \lambda)$ are fitted to experimental macroscopic thermophysical data (e.g. vapor pressures, coexisting liquid-vapor densities, etc.). A unique aspect of the SAFT- $\gamma$ Mie EoS is the accuracy with which the underlying molecular model is the basis of the CG model. The parameters fitted employing the EoS can be used directly in a molecular simulation employing the force field implicit in Eq. 2. This "top-down" fitting procedure produces effective potential parameters which show excellent transferability, representability, and robustness ${ }^{51,57,93}$, being a poor structural accuracy the main issue of this approach ${ }^{62}$. In the case of molecules modeled as homonuclear chains, a faster but equally reliable method to obtain the molecular parameters is the use of corresponding-states correlations, as described in Ref. 52, where experimental critical temperature, a liquid density, and the acentric factor are related to each of the molecular parameters via the SAFT- $\gamma$ Mie EoS.

In molecular models where a ring-like arrangement of the segments is a more accurate description than the linear chain, the SAFT description is at odds with the molecular simulation results. This is discussed in Ref. 63, where the non-linear connectivity of the molecular segments is explicitly considered in the chain term of the SAFT-VR Mie EoS.

We model the small PAHs depicted in Figure 1 ignoring the difference between aromatic and non-aromatic cycles, and represent all of the CG units within a molecule as identical tangentially joined segments segments. We follow Refs. 52,84,94 to model benzene as a monomer, naphthalene as a dimer, and anthracene as a linearly-connected trimer. The CG models for the non-linear structures, pyrene and coronene, are taken from Ref. 63. The Mie parameters are given in Table 1, where, in the last column, the approximate mean-field energies per carbon are given. To obtain those values, we calculate the mean-field energy per CG segment, $\alpha_{m}$, defined as

$$
\begin{aligned}
\alpha_{m} & =-2 \pi \int u(r) r^{2} d r \\
& =2 \pi \epsilon \sigma^{3}\left(\frac{\lambda}{\lambda-6}\right)\left(\frac{\lambda}{6}\right)^{\frac{6}{\lambda-6}}\left(\frac{1}{3}-\frac{1}{\lambda-3}\right),
\end{aligned}
$$


and approximate the total mean-field energy of the molecule as $\alpha_{\text {tot }} \approx m \alpha_{m}$. Furthermore, we calculate the contribution per carbon in our CG representation as $\alpha=\alpha_{\text {tot }} / N_{\text {Ctot }}$, being $N_{\text {Ctot }}$ the total number of carbons in the molecule.

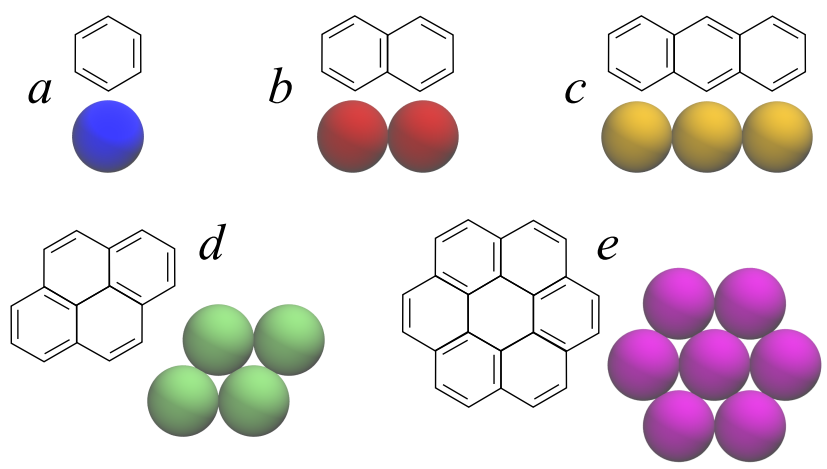

Figure 1: CG representation of $(a)$ benzene, $(b)$ naphthalene, $(c)$ anthracene, $(d)$ pyrene and (e) coronene.

Table 1: CG model parameters for some aromatic molecules estimated via the SAFT- $\gamma$ EoS $^{52}$ and the modified SAFT-VR version of Ref. 63 The last column corresponds to the mean-field energy in our CG representation divided by the number of carbons in the molecule $\left(\alpha=m \alpha_{m} / N_{\text {Ctot }}\right)$.

\begin{tabular}{lccccc}
\hline Molecule & $m$ & $\lambda$ & $\epsilon / k_{\mathrm{B}}[\mathrm{K}]$ & $\sigma[\AA]$ & $\alpha /\left(2 \pi k_{\mathrm{B}}\right)\left[\mathrm{K}^{3}\right]$ \\
\hline Benzene & 1 & 43.81 & 685.62 & 5.33 & 8488.17 \\
Naphthalene & 2 & 19.50 & 557.75 & 4.62 & 7316.93 \\
Anthracene & 3 & 20.76 & 584.83 & 4.38 & 6795.87 \\
Pyrene & 4 & 15.79 & 459.04 & 4.13 & 6037.25 \\
Coronene & 7 & 11.43 & 347.02 & 3.52 & 4080.59 \\
\hline
\end{tabular}

We observe that the absolute values of force-field parameters decrease as the structure becomes more complex. This is in qualitative agreement with the CG models developed by von Lilienfeld and Andrienko ${ }^{44}$, via quantum mechanical density functional theory (DFT), where a monotonous decrease is observed in the Lennard-Jones parameters. Thereupon, it becomes evident that the use of a unique CG unit to build PAHs is not suitable. In the Supporting Information (SI), we provide examples of the poor performance of CG PAHs models which only consider a CG benzene (represented as a monomer) as the building block. 


\section{Trends in PAHs parameters}

To track trends in the PAHs parameters obtained via the SAFT EoS, we calculate the ratio between the number of internal carbons (those that are not linked to hydrogen) $N_{\text {Cint }}$ and the total number of carbons, $N_{\mathrm{Ctot}}$, and use it as a surrogate to represent the molecular morphology. In Figure 2(a), we plot the parameters of Table 1, normalized with respect to the corresponding values for benzene, as a function of the $N_{\text {Cint }} / N_{\text {Ctot }}$ ratio. We propose simple fittings of the parameters to relate values of the $N_{\text {Cint }} / N_{\text {Ctot }}$ ratio with a set of Miepotential parameters and develop CG models for the aromatic cores in asphaltenes. Since the mean-field energy is smoother than the energy parameter, we fit exponential functions to the normalized cubic diameters, repulsive exponents, and mean-field energy, and then, use Equation 3 to obtain the energy parameter. The fitting functions are of the form:

$$
y(x)=y_{0}+y_{1} \exp \left(\frac{x}{y_{2}}\right)
$$

where the variable $x$ is $N_{\text {Cint }} / N_{\text {Ctot }}$, and $y_{0}, y_{1}$, and $y_{2}$ are fitting parameters. The resulting curves are presented in Fig. 2(b). The root-mean-squared error (RMSE) in the fittings are $2.5 \%$ for the diameters, $6.7 \%$ for the repulsive exponents, and $1.7 \%$ for the mean-field energy.

The procedure we propose to obtain CG parameters for polycyclic hydrocarbon structures composed of $m$ fused rings is as follows:

1. Given an arbitrary PAH structure, calculate the ratio between the number of internal carbons and the total number of carbons, $x=N_{\text {Cint }} / N_{\text {Ctot }}$.

2. Calculate the repulsive exponent, diameter, and the mean-field energy per CG segment 

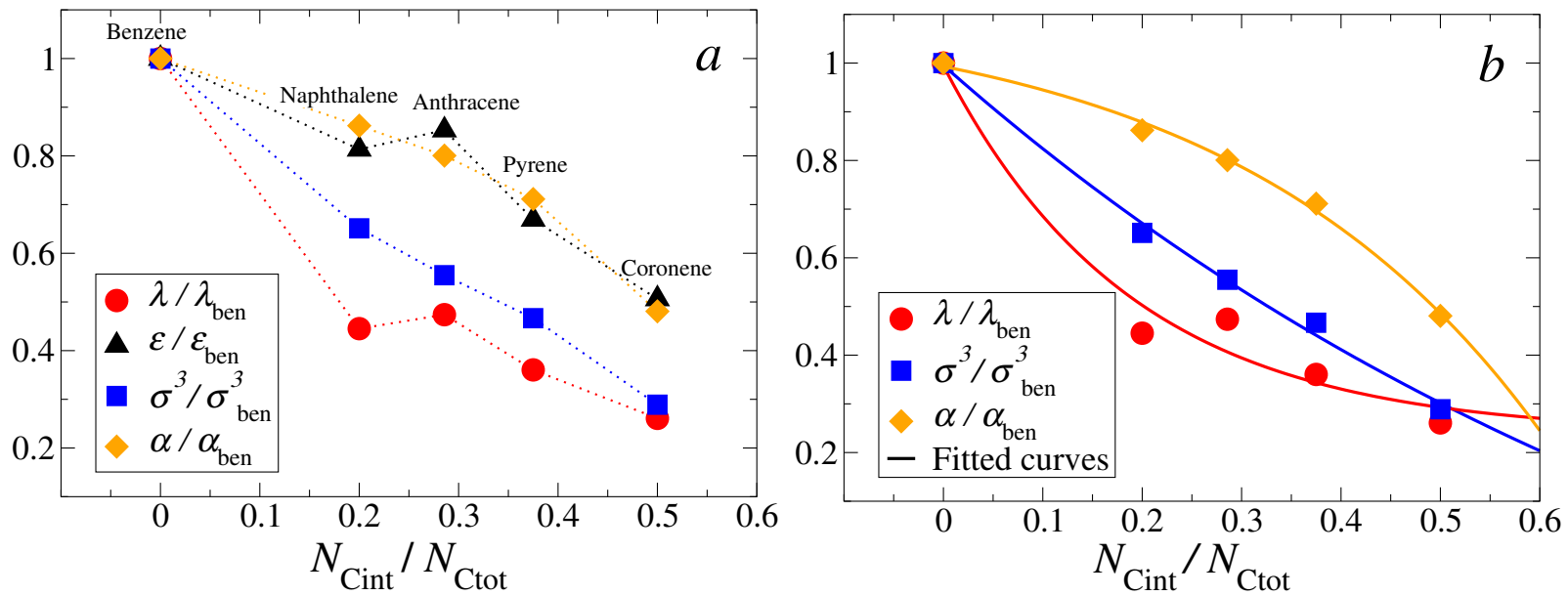

Figure 2: (a) SAFT parameters for CG PAHs plotted as a function of the $N_{\text {Cint }} / N_{\text {Ctot }}$ ratio and normalized respect to benzene parameters. The $N_{\text {Cint }} / N_{\text {Ctot }}$ ratio is 0 for benzene, 0.2 for naphthalene, 0.2857 for anthracene, 0.3750 for pyrene, and 0.5 for coronene. (b) Fitting curves (continuous lines) to the normalized repulsive exponent, $\lambda / \lambda_{\text {ben }}$, cubic diameter, $\sigma^{3} / \sigma_{\text {ben }}^{3}$, and mean-field energy per carbon in the molecule, $\alpha / \alpha_{\text {ben }}$.

using the following relations:

$$
\begin{aligned}
\lambda & =10.42+33.16 \exp \left(-\frac{x}{0.1903}\right) \\
\sigma^{3} & =-95.21+245.9 \exp \left(-\frac{x}{0.8977}\right)^{3} \\
\frac{\alpha_{m}}{2 \pi k_{\mathrm{B}}} & =\left(\frac{N_{\mathrm{Ctot}}}{m}\right)\left[9505-1076 \exp \left(\frac{x}{0.3106}\right)\right] \mathrm{K}^{3},
\end{aligned}
$$

3. Obtain the energy parameter by substituting the previous values in

$$
\frac{\epsilon}{k_{\mathrm{B}}}=\left(\frac{\alpha_{m}}{2 \pi k_{\mathrm{B}}}\right) \frac{1}{\sigma^{3} f(\lambda)}
$$

where

$$
f(\lambda)=\left(\frac{\lambda}{\lambda-6}\right)\left(\frac{\lambda}{6}\right)^{\frac{6}{\lambda-6}}\left(\frac{1}{3}-\frac{1}{\lambda-3}\right)
$$

Following this approach, we obtain molecular parameters for the same cases presented in Table 1, the resulting force fields are given in Table 2. We use SAFT- $\gamma$ Mie EoS and MD simulations to test the performance of this set of parameters and compare it with that of the 
original SAFT parameters. The relative errors for the liquid densities increase from $1 \%$ to $3 \%$ for the linear models and from $3 \%$ to $10 \%$ for the ring-like structures as compared to the results obtained by using the optimal SAFT parameters (Table 1). The details of these tests and resulting plots are given in the SI. An obvious limitation is the modeling of very large PAHs where the mean-field energy for an atomistic carbon model ${ }^{95}$, commonly used for graphene and graphite systems, is reached in our relations for $N_{\text {Cint }} / N_{\text {Ctot }} \approx 0.64$. Still, the models are reliable to predict general trends in the phase diagram of most commonly encountered PAHs.

Table 2: CG model parameters for aromatic molecules calculated by the proposed relations, Equations 5 to 9 . The last column corresponds to the mean-field energy in our CG representation divided by the number of carbons in the molecule $\left(\alpha=m \alpha_{m} / N_{\text {Ctot }}\right)$.

\begin{tabular}{lccccc}
\hline Molecule & $m$ & $\lambda$ & $\epsilon / k_{\mathrm{B}}[\mathrm{K}]$ & $\sigma[\AA]$ & $\alpha /\left(2 \pi k_{\mathrm{B}}\right)\left[\mathrm{K} \AA^{3}\right]$ \\
\hline Benzene & 1 & 43.59 & 683.00 & 5.32 & 8428.92 \\
Naphthalene & 2 & 22.02 & 584.23 & 4.66 & 7456.18 \\
Anthracene & 3 & 17.81 & 544.76 & 4.37 & 6805.04 \\
Pyrene & 4 & 15.05 & 462.00 & 4.06 & 5905.81 \\
Coronene & 7 & 12.82 & 364.49 & 3.58 & 4122.42 \\
\hline
\end{tabular}

\section{CG models}

Two asphaltene models from the literature ${ }^{28,29}$ are studied in this work: the continental "asphaltene C" (AsphC) and the archipelago-like "asphaltene A" (AsphA), with molecular structures depicted in Fig. 3(a) and (b), respectively. We adopt a group-contribution approach to building the CG heteronuclear structures using SAFT- $\gamma$ force fields from simpler molecules to model the non-bonded interactions, and complement the "top-down" approach by tuning flexibility and unlike interactions using information from fully-atomistic simulations. The CG versions of asphaltene $\mathrm{C}$ and $\mathrm{A}$, as well as the $\mathrm{CG}$ solvents (toluene and heptane), are shown in Fig. 4. The aromatic cores are treated as independent structures (cf. Fig. 3(c) and 3(d)) to obtain the CG parameters following the procedure of the previous sec- 
tion. For the AsphC core, we calculate the ratio $x=N_{\text {Cint }} / N_{\text {Ctot }}=20 / 35=0.5714$ and use Equations 5 to 9 to represent it as a homonuclear structure of $m=11$ segments, which are labelled as ARC in Fig. 4(a). Similarly, for AsphA we have $x=N_{\text {Cint }} / N_{\text {Ctot }}=14 / 31=0.4516$ to obtain the Mie potential for the $m=8$ core, with segments labelled as ARA in Fig. 4(b). The 3-segment model for toluene is taken from Ref. 63, where the triangle-like connectivity is explicitly considered. The parameters of the remaining segments are obtained using corresponding-states correlations via the SAFT- $\gamma$ Mie EoS ${ }^{52,84,94}$. For example, the segment labelled as IC5 is obtained by coarse-graining isopentane as a dimer, i.e. using its critical temperature, its saturated liquid density at a temperature of $70 \%$ of the critical one, and the acentric factor from reference data sources ${ }^{96,97}$ to obtain the Mie parameters for a linear chain with $m=2$ using the "Bottled SAFT" web-app ${ }^{84}$. The full list of segment types and the corresponding force-field parameters are given in Table 3, where, in the last column, we include the parent molecule used to obtain the force fields.

It has been observed ${ }^{98,99}$ that the CG heteronuclear models that are built in a groupcontribution approach overestimate the sizes of the represented molecule. To mitigate that effect, we intentionally obtain CG parameters from smaller molecules than the actual moieties identified in the molecular structure. For example, in the AsphC we use parameters for isopentane instead of isohexane in one of the branches, while in the AsphA we use only one segment of the $\mathrm{CG}$ furan that was modeled as a dimer. Another criterion adopted in the selection of the CG parent molecule is the use of CG force fields with repulsive exponents not larger than 40, which appear commonly when modeling heteroatoms, to avoid undesired impetus in the MD simulations. We also try to keep the CG models as simple as possible by using the same type of segments, when appropriate, in different branches of the molecule. We recognize the degeneracy of this procedure, as we could devise several apparently equivalent parametrizations of a macromolecule, hence the need to validate the final model against some property of the underlying molecule. The combining rules that are used to evaluate 


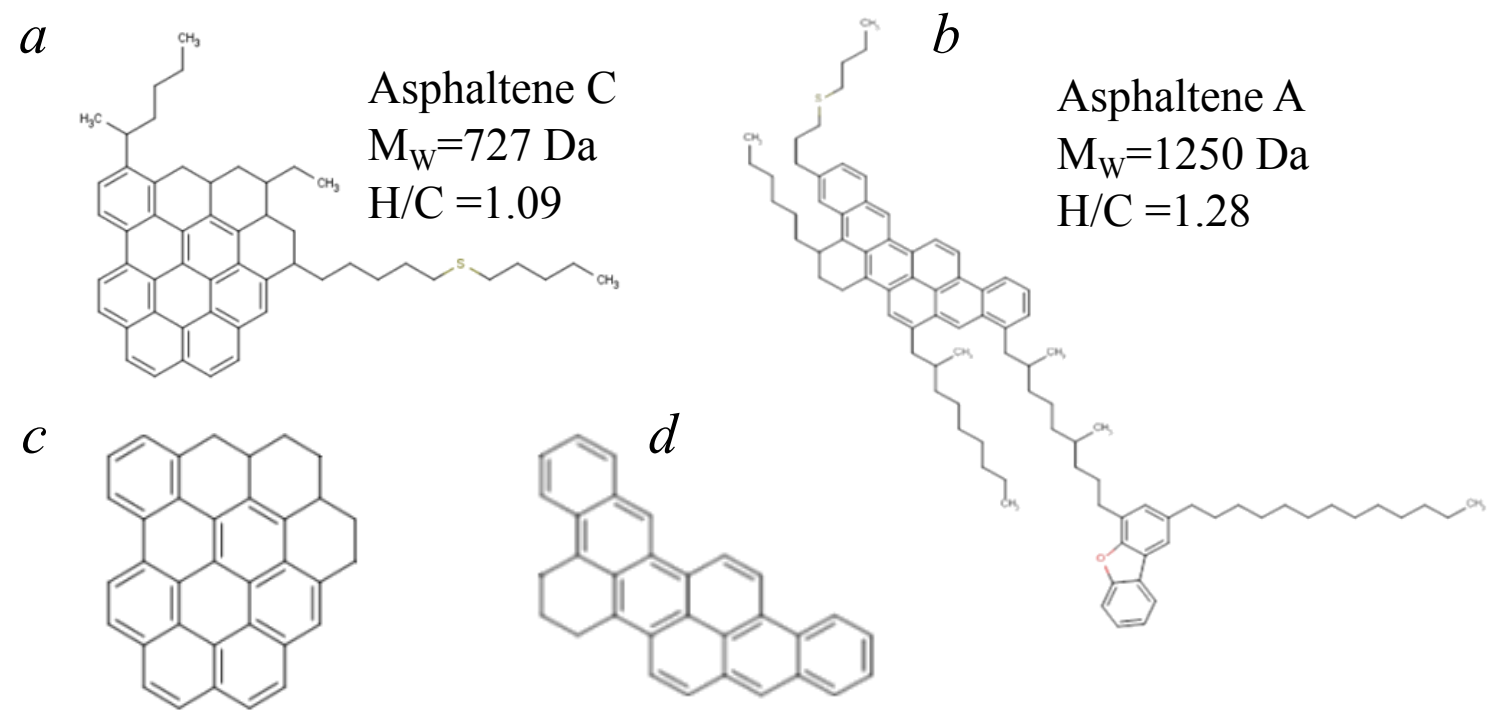

Figure 3: Molecular structures for $(a)$ asphaltene $\mathrm{C}$ and $(b)$ asphaltene $\mathrm{A}^{28,29}$. The polycyclic cores of both asphaltenes are presented as independent structures in $(c)$ and $(d)$.
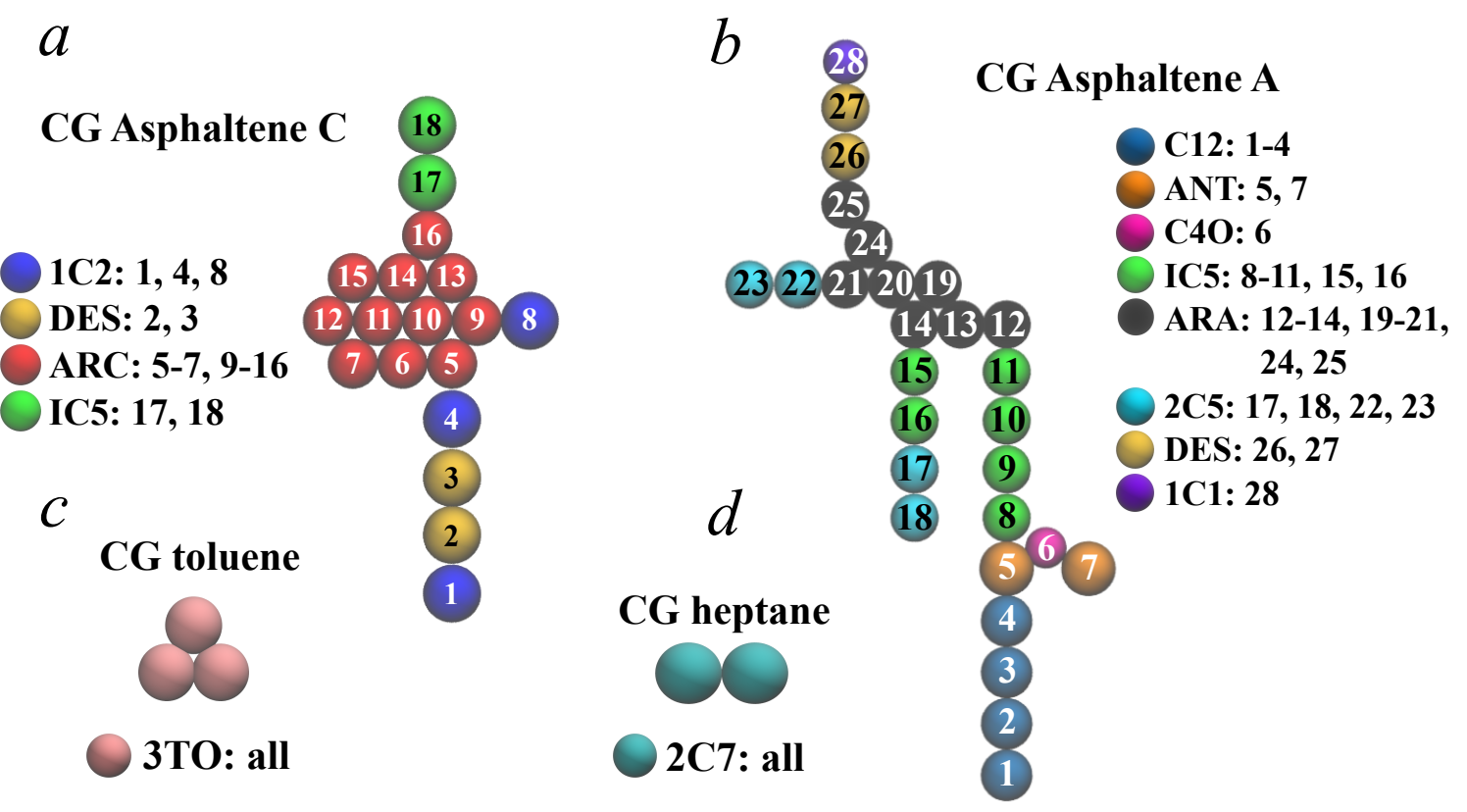

Figure 4: Molecular models in our CG systems: (a) asphaltene C, $(b)$ asphaltene A, $(c)$ toluene, and $(d)$ heptane. The name given to each type of segment and the corresponding number in the model is also shown. 
Table 3: Mie force fields for CG asphaltenes, toluene, and heptane. The name for each segment corresponds to the lists in Fig. 4. We use the procedure presented in this work to parametrize the aromatic-core segments, labelled as "ARC" and "ARA".

\begin{tabular}{|c|c|c|c|c|}
\hline Segment & $\lambda$ & $\epsilon / k_{\mathrm{B}}[\mathrm{K}]$ & $\sigma[\AA]$ & Parent molecule \\
\hline \multicolumn{5}{|c|}{ Asphaltenes } \\
\hline $\mathrm{ARC}$ & 12.07 & 281.13 & 3.268 & Fig. $3 c(m=11)$ \\
\hline ARA & 13.52 & 433.10 & 3.768 & Fig. $3 d(m=8)$ \\
\hline ANT & 20.76 & 584.83 & 4.380 & Anthrace $(m=2)^{a}$ \\
\hline $1 \mathrm{C} 1$ & 16.39 & 170.75 & 3.752 & Methane $(m=1)^{b}$ \\
\hline $1 \mathrm{C} 2$ & 27.30 & 330.25 & 4.349 & Ethane $(m=1)^{b}$ \\
\hline $2 \mathrm{C} 5$ & 16.06 & 317.50 & 4.248 & Pentane $(m=2)^{b}$ \\
\hline $\mathrm{C} 12$ & 18.41 & 378.56 & 4.351 & Dodecane $(m=4)^{b}$ \\
\hline IC5 & 14.92 & 298.38 & 4.236 & Isopentane $(m=2)^{b}$ \\
\hline DES & 18.99 & 410.47 & 4.278 & Diethyl sulphide $(m=2)^{a}$ \\
\hline $\mathrm{C} 4 \mathrm{O}$ & 14.25 & 308.93 & 3.649 & Furan $(m=2)^{a}$ \\
\hline \multicolumn{5}{|l|}{ Toluene } \\
\hline $3 \mathrm{TO}$ & 11.80 & 268.24 & 3.685 & Toluene $(m=3)^{c}$ \\
\hline \multicolumn{5}{|l|}{ Heptane } \\
\hline $2 \mathrm{C} 7$ & 23.81 & 436.13 & 4.766 & Heptane $(m=2)^{b}$ \\
\hline
\end{tabular}

${ }^{a}$ Parameters obtained following Refs. 52,84. ${ }^{b}$ Parameters taken from Ref. 94. ${ }^{c}$ Parameters taken from Ref. 63, explicitly considering the ring-like structure. 
interactions between two unlike segments $i$ and $j$ are:

$$
\sigma_{i j}=\frac{\sigma_{i i}+\sigma_{j j}}{2}
$$

which is the Lorentz rule for the diameters,

$$
\lambda_{i j}-3=\left[\left(\lambda_{i i}-3\right)\left(\lambda_{j j}-3\right)\right]^{1 / 2},
$$

for the repulsive exponent ${ }^{98}$, and

$$
\epsilon_{i j}=\left(1-k_{i j}\right) \frac{\sqrt{\sigma_{i i}^{3} \sigma_{j j}^{3}}}{\sigma_{i j}^{3}} \sqrt{\epsilon_{i i} \epsilon_{j j}}
$$

for the energy ${ }^{98}$, where the binary adjustable parameter $k_{i j}$ allows us to improve the description of unlike interactions that strongly deviate from the above combination rules. We use a value of $k_{i j}=-0.22$ to take into account the strong interactions between heteroatoms and aromatic segments in asphaltenes. The selection process for this $k_{i j}$ value is summarized in the CG model validation section, where AsphC systems are used to tune the ARC-DES interactions. For the AsphA systems, the unlike-interaction value $k_{i j}=-0.22$ is transferred to model the following pair interactions: ARA-C4O, ARA-DES, ANT-C4O, and ANT-DES. We assume that this unlike-interaction parameter is temperature independent, as observed in other studies ${ }^{99}$. All the other remaining unlike-interaction energies are calculated from Eq. 12 with $k_{i j}=0$.

The contiguous segments in SAFT CG models are tangentially bonded, which is implemented in the MD simulations by a harmonic-spring potential, $V_{\mathrm{b}}=\left(k_{\mathrm{b}} / 2\right)\left(r-r_{0}\right)^{2}$, with a very stiff spring constant of $k_{\mathrm{b}}=2 \times 10^{4} \mathrm{~kJ} \mathrm{~mol}^{-1} \mathrm{~nm}^{-2}$, and the equilibrium position $r_{0}$ taken at the value of the corresponding diameter, $\sigma_{i j}$. Angular restrictions are modeled by harmonic potentials of the form $V_{\mathrm{a}}=\left(k_{\mathrm{a}} / 2\right)\left(\theta-\theta_{0}\right)^{2}$. For the aliphatic branches, we use a set of parameters from the CG alkanes representation detailed in Ref. 57, where the spring 
constant is $k_{\mathrm{a}}=22.18 \mathrm{~kJ} \mathrm{~mol}^{-1} \mathrm{rad}^{-2}$ and the equilibrium angle is $\theta_{0}=157.6^{\circ}$. For the polycyclic cores, we use a spring constant of $k_{\mathrm{a}}=50 \mathrm{~kJ} \mathrm{~mol}^{-1} \mathrm{rad}^{-2}$, which allows the core to have a planar structure but retaining some degree of flexibility. We use AsphC systems to tune this parameter, as presented in the CG model validation section, to match the aggregation properties of the atomistic benchmarks. The spring-constant value is transferred, without further examination, to the AsphA systems. The complete list of bonds and angles in the GROMACS ${ }^{100}$ format is given in the SI for both asphaltene models.

\section{Simulation details}

We study the proposed CG models via classical MD simulations using the GROMACS ${ }^{100}$ software v4.5.5 and v5.1.3. The validation tests are performed using CG systems with 27 asphaltenes (A or C) and explicit solvent (toluene or heptane) at a concentration of $\Phi_{\text {Asph }}=$ $7 \mathrm{wt} \%$, at a temperature of $T=300 \mathrm{~K}$ and at a pressure of $P=1 \mathrm{~atm}$. The conditions are chosen to match those of the fully-atomistic systems presented in Ref. 28. The CG systems are simulated with a potential cutoff of $25 \AA$ and a time step of $\Delta t=0.01 \mathrm{ps}$, which is 10 times larger than that used in the atomistic models. This, on its own improves by an order of magnitude the performance of the CG simulations. The systems are initially equilibrated during $30 \mathrm{~ns}$ in the isothermal-isobaric $(N P T)$ ensemble. Then, having an equilibrated density, we run up to $800 \mathrm{~ns}$ in the canonical $(N V T)$ ensemble. The coupling algorithms are the Nose-Hoover thermostat ${ }^{101,102}$ and the Parrinello-Rahman barostat ${ }^{103,104}$ for NPT simulations. The initial configurations are generated by inserting the molecules at random positions in the simulation box, unless stated otherwise. The wall-clock time required to simulate 27 CG AsphC in heptane during $500 \mathrm{~ns}$ ( $6000 \mathrm{CG}$ segments, using $\Delta t=0.01 \mathrm{ps})$, for example, is around 18 hours, while the same system in the fully atomistic representation $(\sim 60000$ atoms, using $\Delta t=0.001 \mathrm{ps})$ required around 40 days to reach the $500 \mathrm{~ns}$ of simulation, using the same computational resources (24 Intel CPU Xeon CPUs E5-2643 v2 
( $3.50 \mathrm{GHz}$ ). We verify the system equilibrium by monitoring the asphaltene-asphaltene energy as a function of time, given that the total energy is dominated by the solvent and may appear as deceptively stable. The snapshots depicted in this work are produced using $\operatorname{VMD}^{105,106}$.

The changes in the aggregation properties are investigated using AsphC systems by varying asphaltene concentration, solvent composition, and temperature. The list of systems with details of system size and composition is presented in the SI. In these simulations, the last 200 to $250 \mathrm{~ns}$ of equilibrated trajectories are analyzed to quantify the aggregation of molecules and calculate structural properties of clusters. Two criteria are applied to determine if two asphaltenes are classified as part of a cluster:

1. In the first criterion, the pair distances $r_{i j}$ between all segments $i$ in a molecule and all segments $j$ in a second molecule are considered. If one of the $r_{i j}$ distances is lower than a cutoff value, $r_{i j} \leq d_{\text {cut }}$, the molecules are marked as part of the same cluster. This algorithm is used in the validation tests of the CG models (systems with 27 asphaltenes at $7 \mathrm{wt} \%$ ) as it is equivalent to the criterion adopted in Ref. 28 to analyze the fully-atomistic systems to which we are benchmarking to.

2. In the second criterion, the segment-segment distances between two asphaltenes $r_{i j}$ are compared with the cutoff value only if $i$ or $j$ are part of the aromatic cores (the segments labelled as ARC or ARA in Fig. 4). In other words, the branch-branch pairs are excluded in the algorithm. Again, the molecules are marked as part of the same cluster if $r_{i j} \leq d_{\text {cut }}$. This criterion is applied in the analysis of all of the systems presented in the Large-scale simulations section.

The cutoff distance is chosen as $d_{\text {cut }}=4.5 \AA$ in both criteria although it is noted that we obtain similar results with cutoff distances between 4.3 and $4.7 \AA$. In Fig. 5, we present a visual example of the cluster identification algorithm by applying both criteria to the same group of molecules. We see how criterion 1 seems too forgiving, as pointed out in 
Ref. 55, and can generate overestimation of the cluster size, in particular when dealing with high-concentration systems, where casual branch-branch proximity occurs. For comparison, the visualization of the clusters detected with a further restrictive criterion, in which only core-core pairs are considered is also shown. It is apparent from the figure that this latter criterion does not capture the correct cluster distribution.
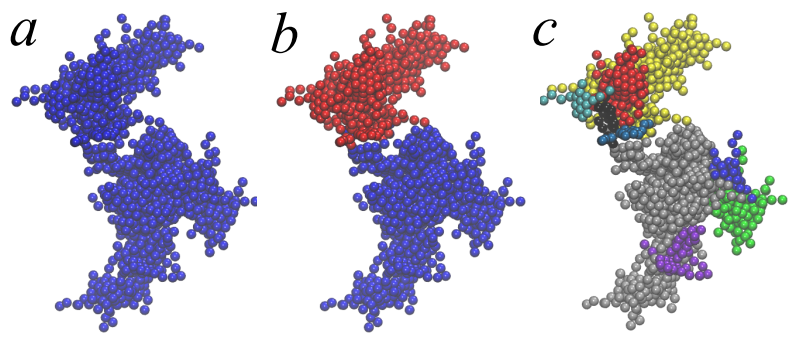

Figure 5: Visualization of the clusters identified using different criteria over the same group of molecules. Every cluster is indicated with a different color. In all algorithms, the distance between segments belonging to different molecules are compared to the same cutoff value, but a different criterion, based on the segment identity, is applied: $(a)$ considering all segmentsegment distances, $(b)$ dismissing the branch-branch pairs, $(c)$ counting only core-core pairs.

The number of structures formed by $n$ molecules (cluster size) is stored through the equilibrium trajectory into histograms that are later averaged over the total number of configurations in the analysis. We define these averages as the occurrence or frequency, $f_{n}$, of clusters of size $n$. The cluster-size probability $P_{n}$ is the probability of finding a molecule in a cluster of size $n$. To obtain it, every frequency $f_{n}$ is multiplied by the corresponding cluster size and divided by the total number of asphaltenes in the system, $n_{\mathrm{Asph}}$

$$
P_{n}=\frac{n f_{n}}{n_{\mathrm{Asph}}}
$$

We report all of the species in the cluster-size histograms, however, for the structural properties, only clusters formed by at least two asphaltenes are analyzed, following the criteria of Ref. 28. To track the time evolution of cluster sizes, for every analyzed configuration, the maximum cluster size $n_{\max }$ is stored as well as the average cluster size per frame $n_{\text {ave }}$, which calculated over structures with $n \geq 2$. The average value, calculated over all $n_{\text {ave }}$ along the 
equilibrium trajectory, is reported as $\bar{n}$. Also, for every cluster with $n \geq 2$, we calculate the radius of gyration $R_{\mathrm{g}}$, the relative-shape-anisotropy factor $\kappa^{228,107}$ (that takes values between 0 and 1, where values close to 0 correspond to clusters with a spherical shape and $\kappa^{2}$ values close to 1 are related to cigar-like aggregates), and the cluster density, following the definitions in Ref. 28. The probability functions for each property $\left(R_{\mathrm{g}}, \kappa^{2}\right.$, and $\left.\rho_{c l u}\right)$ are obtained by averaging the frequency distributions over the total number of clusters analyzed in the equilibrium trajectory. The mathematical expressions and details are given in the SI.

\section{CG model validation}

\section{Fine tuning: flexibility and unlike interactions}

We use the system consisting of 27 AsphC molecules in heptane at $\Phi_{\text {Asph }}=7 \mathrm{wt} \%$, simulated at a temperature of $T=300 \mathrm{~K}$ and at a pressure of $P=1$ bar, to study the effects of the core flexibility in the aggregation properties. In particular, we focus on the cluster shapes, quantified via the relative-shape-anisotropy-factor $\left(\kappa^{2}\right)$ distributions. The target $\kappa^{2}$ distribution from the fully-atomistic simulations, taken from Ref. 28, is presented as a continuous line in Fig. 6. A mono-modal curve is observed with a peak in $\kappa^{2}$ values close to 0.1, which is an indication of quasi-spherical clusters, like the snapshot shown in the inset. The remaining curves and snapshots in Fig. 6 correspond to CG systems with different spring constants, $k_{\mathrm{a}}$, of the angular restrictions of the aromatic cores. In all of the cases, a columnar stacking between neighboring asphaltenes is the dominant feature, however, larger values of $k_{\mathrm{a}}$ (rigid-like core) promote a sharp columnar arrangement in the aggregates, that does not correspond to the type of aggregates formed in the atomistic simulations. We observe this, qualitatively, in the corresponding snapshots and quantitatively in the peaks at large values of the $\kappa^{2}$ distributions. A value of $k_{\mathrm{a}}=50 \mathrm{~kJ} \mathrm{~mol}^{-1} \mathrm{rad}^{-2}$ allows some flexibility to the aromatic core, which enables the aggregation of AsphC in clusters with similar shapes to those of the atomistic benchmark, but at the same time, it is large enough to keep the desired 
planar configuration of the core. The flexibility effects are tested before and after tuning the unlike interactions, the results presented in Fig. 6 (final model) remain true for both cases: long aggregates when the asphaltenes are modeled with highly rigid cores $\left(k_{\mathrm{a}} \geq 75 \mathrm{~kJ} \mathrm{~mol}^{-1}\right.$ $\operatorname{rad}^{-2}$ ), and globular-like aggregates for lower $k_{\mathrm{a}}$ values.

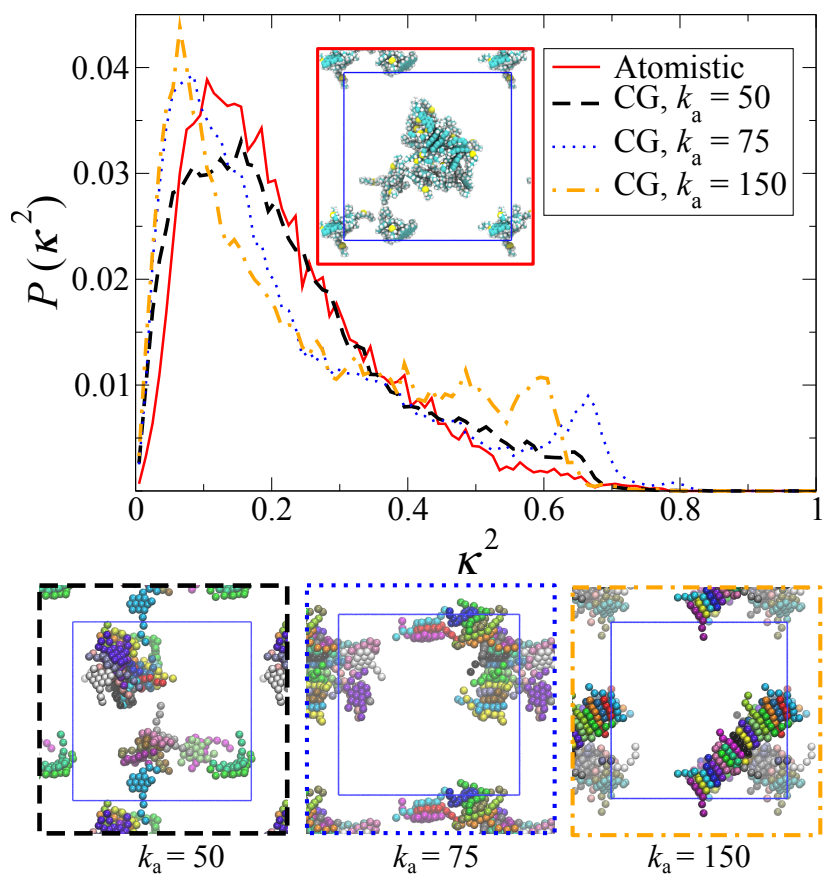

Figure 6: Relative-shape-anisotropy-factor distributions calculated from $27 \mathrm{AsphC}+$ heptane systems. The distribution from fully-atomistic simulations is presented as a continuous red line, while the other curves correspond to CG systems with different spring constants $k_{\mathrm{a}}$ in the angular restrictions of the aromatic cores. The units of $k_{\mathrm{a}}$ are $\mathrm{kJ} \mathrm{mol}{ }^{-1} \mathrm{rad}^{-2}$. In the inset, we show an equilibrium configuration of the target (atomistic) system and at the bottom, typical snapshots of the CG systems simulated with the $k_{\text {a }}$ values considered. The solvent molecules are omitted and each asphaltene is represented in a different color to ease the visualization. The blue frame inside the snapshots represents the simulation box in the periodic systems.

After fixing the flexibility restrictions, we tune the unlike interactions between segments representing heteroatoms and those representing aromatic cores, which corresponds to the ARC-DES pairs in the AsphC system. We use the cluster-size distributions from fullyatomistic 27 AsphC + solvent systems as targets, which are presented as continuous lines in Figs. 7(a) and 7(b), for toluene and heptane, respectively. The remaining lines correspond to the cluster-size distributions from CG systems simulated with different values of the $k_{i j}$ 
parameter, see Eq. 12. For the starting value of $k_{i j}=0$ (not shown) small clusters are predominant in both solvents, which strongly disagrees with the expected behavior. As the ARC-DES attractions are increased (negative values of $k_{i j}$ ), larger clusters are formed in the systems. The CG heptane system with $k_{i j}=-0.25$ presents a good agreement with the atomistic cluster-size distribution (Fig. $7(b)$ ), however, such value generates unrealistically large structures in the toluene system (Fig. $7(a)$ ). We choose $k_{i j}=-0.22$ as a value that reproduces in the best way the expected behavior in both solvents. Degeneracy in the tuned parameters is expected, since a low value of the core spring constant increases the mobility of the molecule and lowers the clustering of the systems, while the negative $k_{i j}$ parameter promotes aggregation. However, we consider that the chosen values reproduce the main features of the molecules and aggregates in the atomistic systems. The flexibility and unlike interactions tuned with AsphC systems are transferred to the AsphA model without further modification.
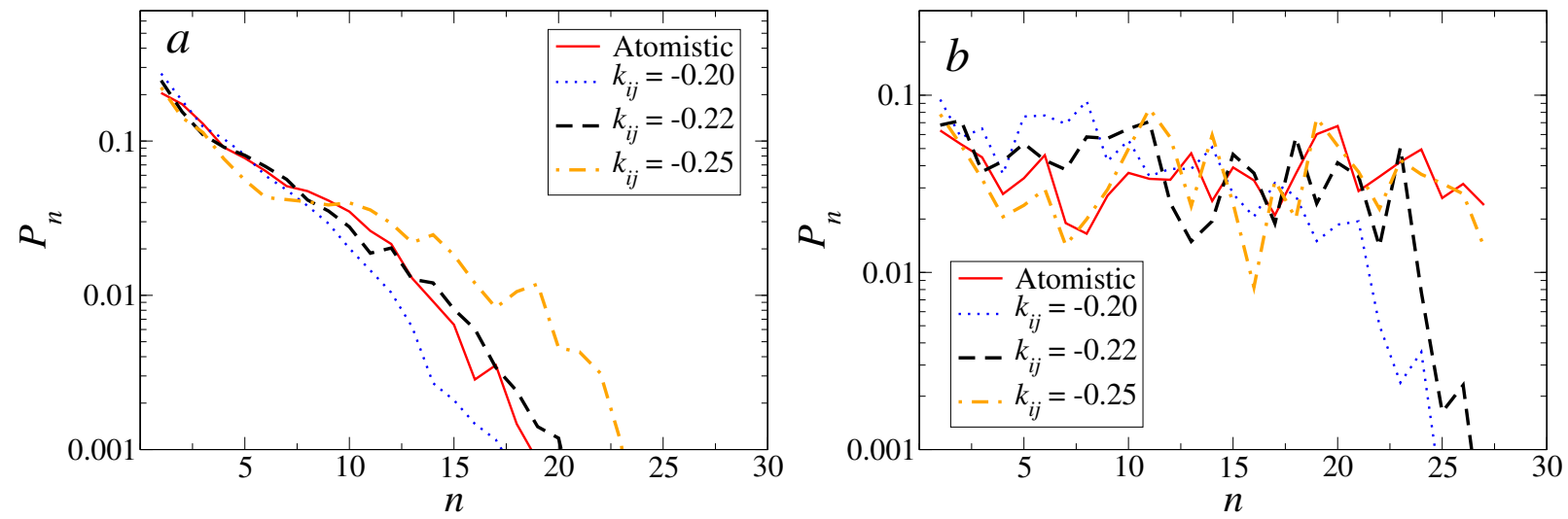

Figure 7: Cluster-size distributions calculated from (a) 27 AsphC + toluene and (b) 27 AsphC + heptane systems. The target distributions from fully-atomistic simulations are presented as continuous lines, while the other histograms correspond to CG systems with different unlike-interaction parameters.

\section{Asphaltene C: comparison to atomistic model}

Further validation of the $\mathrm{CG}$ AsphC model is presented in Figs. 8 and 9. Configurations from the last $250 \mathrm{~ns}$ of simulation, taken every $0.02 \mathrm{~ns}$, are analyzed to obtain the structural 
properties of clusters. The cluster-size probability as a function of the cluster size, $n$, is plotted in Fig. 8(a) and (b) for the toluene and heptane systems, respectively. The continuous lines represent the atomistic benchmarks from Ref. 28 and the dashed lines correspond to the CG results. Both systems of AsphC in toluene have monomers as the predominant structures, the distributions monotonously decay up to $n \sim 20$ molecules. For asphaltene $\mathrm{C}$ in heptane, the cluster-size distributions are broad and it is possible to observe clusters with sizes of the order of the system (27 asphaltenes). The snapshots of equilibrium configurations are shown at the bottom of the figure, where we appreciate an excellent agreement between both levels of description, with small clusters of AsphC in the toluene systems and larger aggregates of AsphC in heptane. A quantitative analysis of the structures with $n \geq 2$ is shown in Fig. 9. In Fig. 9(a) and (b), the average cluster size $n_{\text {ave }}$ as a function of time for 500 ns of simulation is shown for atomistic and CG systems, respectively. The ensemble average cluster sizes are $\langle n\rangle=4.1 \pm 1.2$ and $10.6 \pm 5.7$ molecules for the atomistic toluene and heptane systems, while those for the CG systems are $\langle n\rangle=4.3 \pm 1.5$ and $8.0 \pm 3.1$ molecules. With the same color code of Fig. 8, we present the radius of gyration $R_{\mathrm{g}}$, relative-shape-anisotropy factor $\kappa^{2}$, and cluster density $\rho_{\text {clu }}$ distributions in Figs. $9(c)-(e)$. The cluster-size and $\kappa^{2}$ distributions were used to tune flexibility and unlike interactions of the model, as discussed in the previous section, hence, the quantitative agreement observed therein is expected. In the $R_{\mathrm{g}}$ histograms, the position of the main peak predicted in the CG systems matches the atomistic benchmarks, with similar features in both representations. The CG curves for the $\rho_{\text {clu }}$ probabilities have the correct trend for each solvent, however, that of the heptane system is shifted with respect to the atomistic histogram, indicating that the CG clusters in heptane are denser. Overall, the agreement between both sets of simulations is excellent.

\section{Asphaltene A: comparison to atomistic model}

In a test of the transferability of the force field, we employ the model potential parameters obtained from AsphC systems directly to predict the properties of the unrelated AsphA 


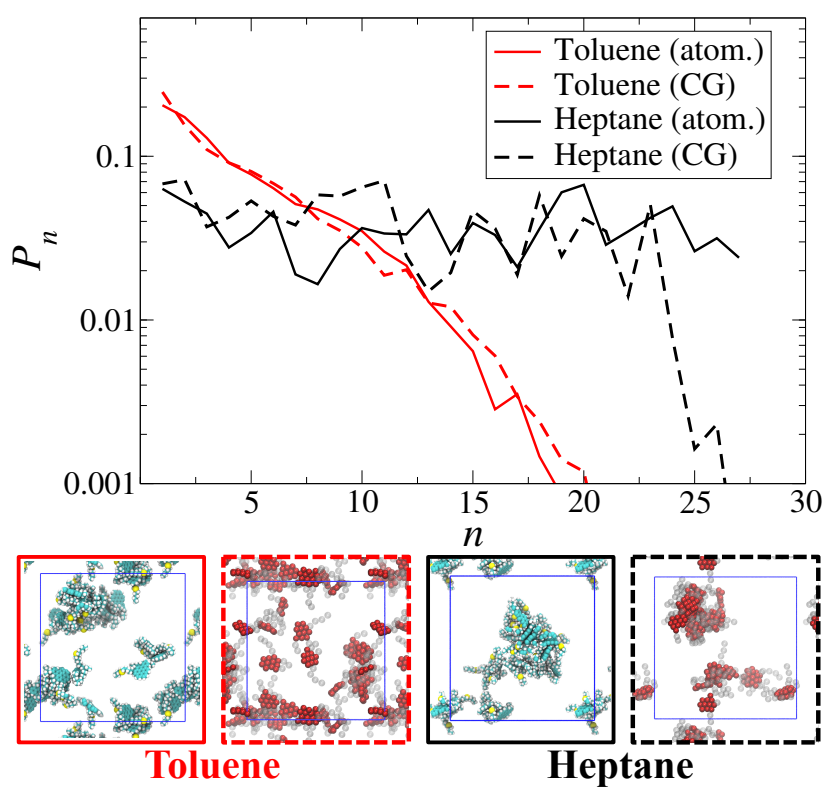

Figure 8: Cluster-size probabilities and snapshots of equilibrated configurations from atomistic (solid line) and CG (dashed line) systems with $27 \mathrm{AsphC}$ molecules in (a) toluene (red) and (b) heptane (black). The atomistic results are taken from Ref. 28. The solvent molecules are omitted to ease the visualization, the CG cores are shown in a solid color and the branches are represented in a transparent grey.

model. The direct comparison of the CG AsphA systems and the fully-atomistic results from Ref. 28 are presented in Figs. 10 and 11, with the same layout and color code as that of Figs. 8 and 9. The similarity in aggregation behavior of this model asphaltene in both solvents, as can be appreciated in the cluster-size histogram (Fig. 10) and in the $n_{\text {ave }}$ plot (Fig. 11(a) and $(b)$ ), makes this test particularly challenging, however, the CG model is able to match the target cluster-size distributions. A qualitative agreement is also observed in the snapshots of the CG and atomistic systems (Fig. 10), with diffuse clusters in toluene and in heptane. The ensamble average cluster sizes from the atomistic benchmarks are $\langle n\rangle=5.7 \pm 2.5$ and $6.7 \pm 2.8$ in the toluene and heptane systems, while the corresponding ensemble averages are $\langle n\rangle=5.7 \pm 3.0$ and $7.2 \pm 3.8$ in the $\mathrm{CG}$ representation. The $R_{\mathrm{g}}$ histograms in the CG systems are shifted to larger values (by around $3 \AA$ ) with respect to the atomistic benchmarks, presumably due to the bulky representation of the CG model and/or an effect of flexibility that is not taken into account. The relative-shape-anisotropy parameter is correctly predicted in the CG systems, where the small differences in the trends 

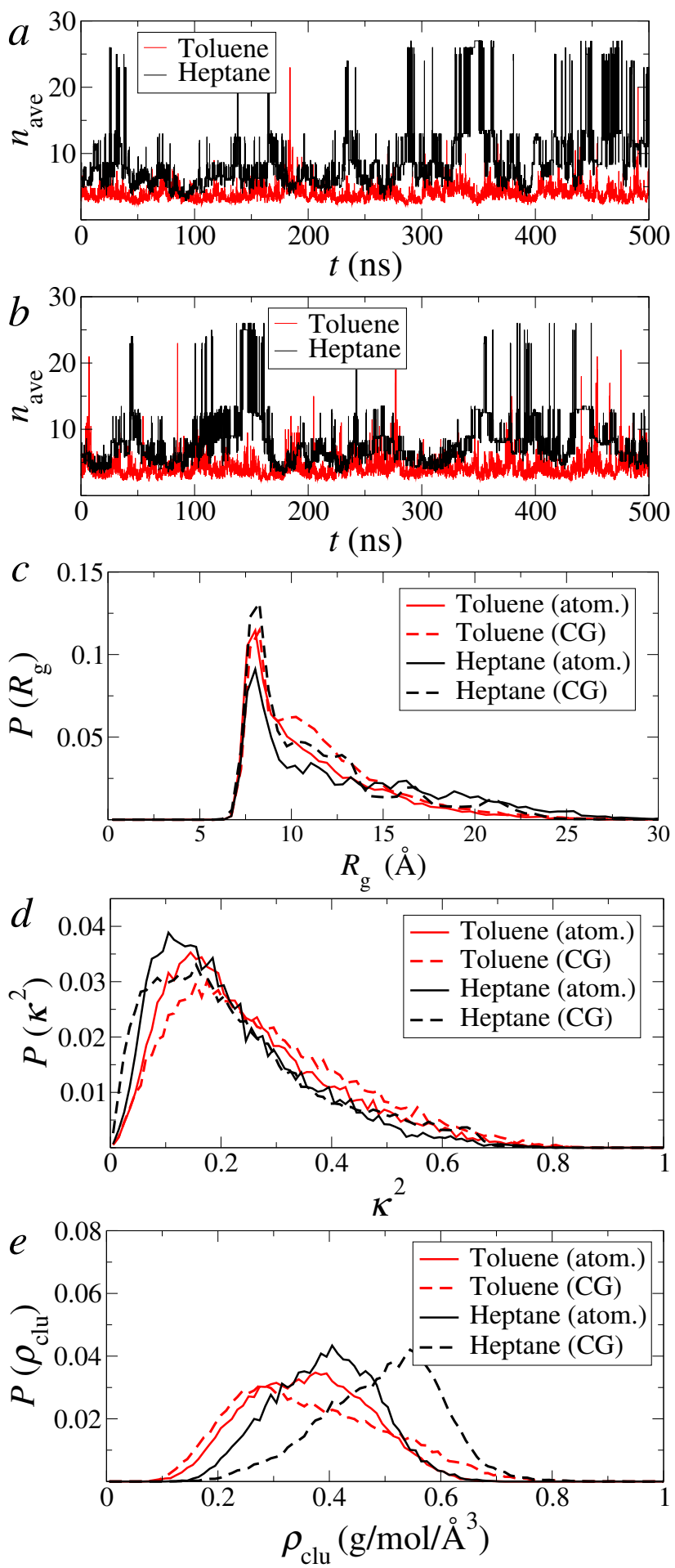

Figure 9: Structural analysis of clusters $(n \geq 2)$ from MD simulations of 27 AsphC molecules in explicit toluene (red) or heptane (black). Average cluster-size, $n_{\text {ave }}$ as a function of time from $(a)$ the fully-atomistic systems in Ref. 28 , and $(b)$ the CG simulations in this work. Probability distributions are for the $(c)$ radius of gyration, $R_{\mathrm{g}},(d)$ relative-shape-anisotropy factor, $\kappa^{2}$, and $(e)$ cluster density, $\rho_{\text {clu }}$. The continuous lines correspond to results from atomistic simulations and dashed lines to results from CG simulations. 
of both solvents are captured. Some differences are observed for the density distributions, where the CG results are shifted to the left, with respect to the benchmarks, in both solvents. This quantitative disagreement could be an effect of the overestimated radius of gyration in the CG aggregates, that would propagate as a power of -3 in the density calculations. The trend in the $\rho_{\text {clu }}$ histograms is otherwise correct, with denser aggregates in heptane than in toluene. In summary, the structural cluster properties predicted in the CG AsphA systems are a fair representation of the aggregation exhibited in fully-atomistic systems.

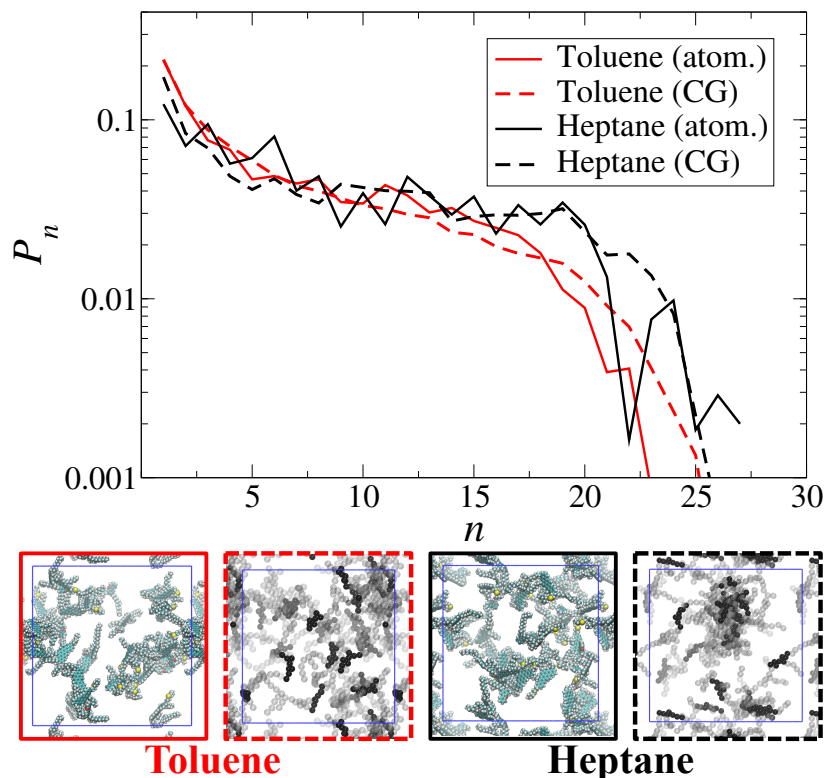

Figure 10: Cluster-size probabilities and snapshots of equilibrated configurations from atomistic (solid line) and CG (dashed line) systems with 27 AsphA molecules in (a) toluene (red) and $(b)$ heptane (black). The atomistic results are taken from Ref. 28. The solvent molecules are omitted to ease the visualization, the CG cores are shown in a solid color and the branches are represented in a transparent grey.

\section{Large-scale simulations}

\section{Aggregation trends}

Small simulations (of the order of dozens of asphaltenes), while useful to study the incipient stages of aggregation, can provide a misleading physical picture as they are inherently 

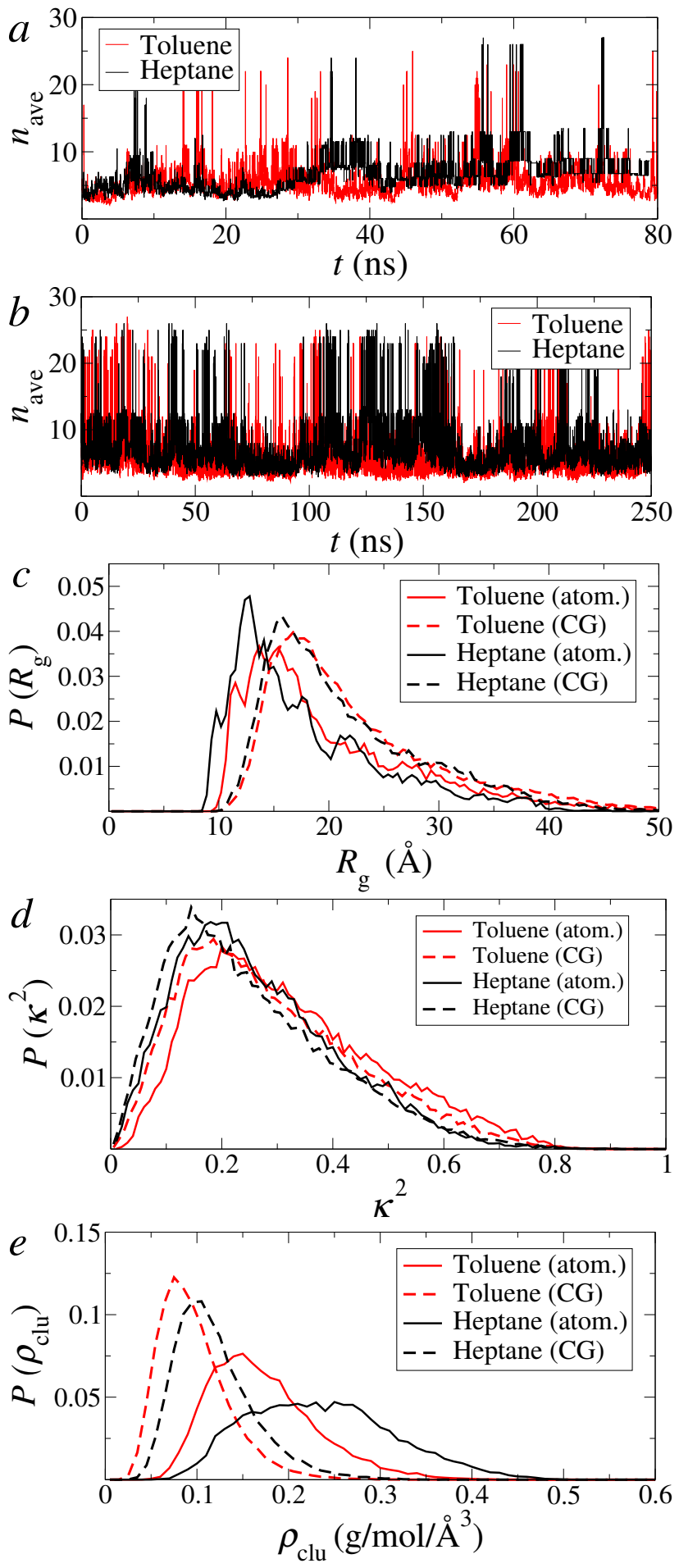

Figure 11: Structural analysis of clusters $(n \geq 2)$ from MD simulations of 27 AsphA molecules in explicit toluene (red) or heptane (black). Average cluster-size, $n_{\text {ave }}$ as a function of time from $(a)$ the fully-atomistic systems in Ref. 28, and (b) the CG simulations in this work. Probability distributions are for the $(c)$ radius of gyration, $R_{\mathrm{g}},(d)$ relative-shape-anisotropy factor, $\kappa^{2}$, and $(e)$ cluster density, $\rho_{\text {clu }}$. The continuous lines correspond to results from atomistic simulations and dashed lines to results from CG simulations. 
incapable of discerning between the formation of large and stable clusters and a full twophase separation, and will typically only explore inadequate short times. We exploit the capacity of the CG models to accurately describe the thermodynamics of asphaltenic systems to quantify in a detailed fashion the aggregation behavior of our models at different state conditions. Aggregation is quantified via cluster-size histograms, which are calculated from the last 200 ns of simulation, excluding branch-branch contacts in the cluster criterion, as explained in the Simulation details section. The cluster-size probabilities obtained from series of asphaltene concentration, solvent composition, and temperature are presented in Figs. $12(a),(b)$, and $(c)$, respectively. The asphaltene concentration effect is studied with simulation boxes of 216 AsphC molecules in explicit heptane at asphaltene concentration of $\Phi_{\mathrm{Asph}}=3,7,15,20$, and $25 \mathrm{wt} \%$, at a temperature of $300 \mathrm{~K}$, and at a pressure of 1 bar. To study the effect of the solvent composition, we simulate systems with 216 AsphC molecules at $\Phi_{\text {Asph }}=7 \mathrm{wt} \%$ in toluene and heptane mixtures with toluene compositions of $x_{\mathrm{tol}}=100$, $75,50,25$, and 0 (wt/wt $\%$ ), at $T=300 \mathrm{~K}$, and $P=1$ bar. The effect of temperature in the aggregation is tested using systems with 504 AsphC molecules in explicit heptane at an asphaltene concentration of $10 \mathrm{wt} \%$ and at a pressure of 1 bar. The temperatures simulated are $T=450,350300,275$, and $250 \mathrm{~K}$. In the insets of Fig. 12, the details of some histograms are shown. Further details are given in the SI.

We summarize the aggregation trends observed with the schematic representation of Fig. 13, where the systems change from low aggregation conditions (curves $a-c$ ) towards strong clustering $(e)$ as the asphaltene concentration is increased, the toluene amount in the solvent is decreased or as the temperature is lowered, passing through intermediate states with histograms characterized by the rise of a long tail or shoulder at large values of $n(d)$. In the next subsections, the reliability of these numerical results is challenged by simulating systems with up to 2000 AsphC molecules to test system-size, reversibility, and time dependence on three exemplary histograms, selected in correspondence with the three levels of aggregation. 

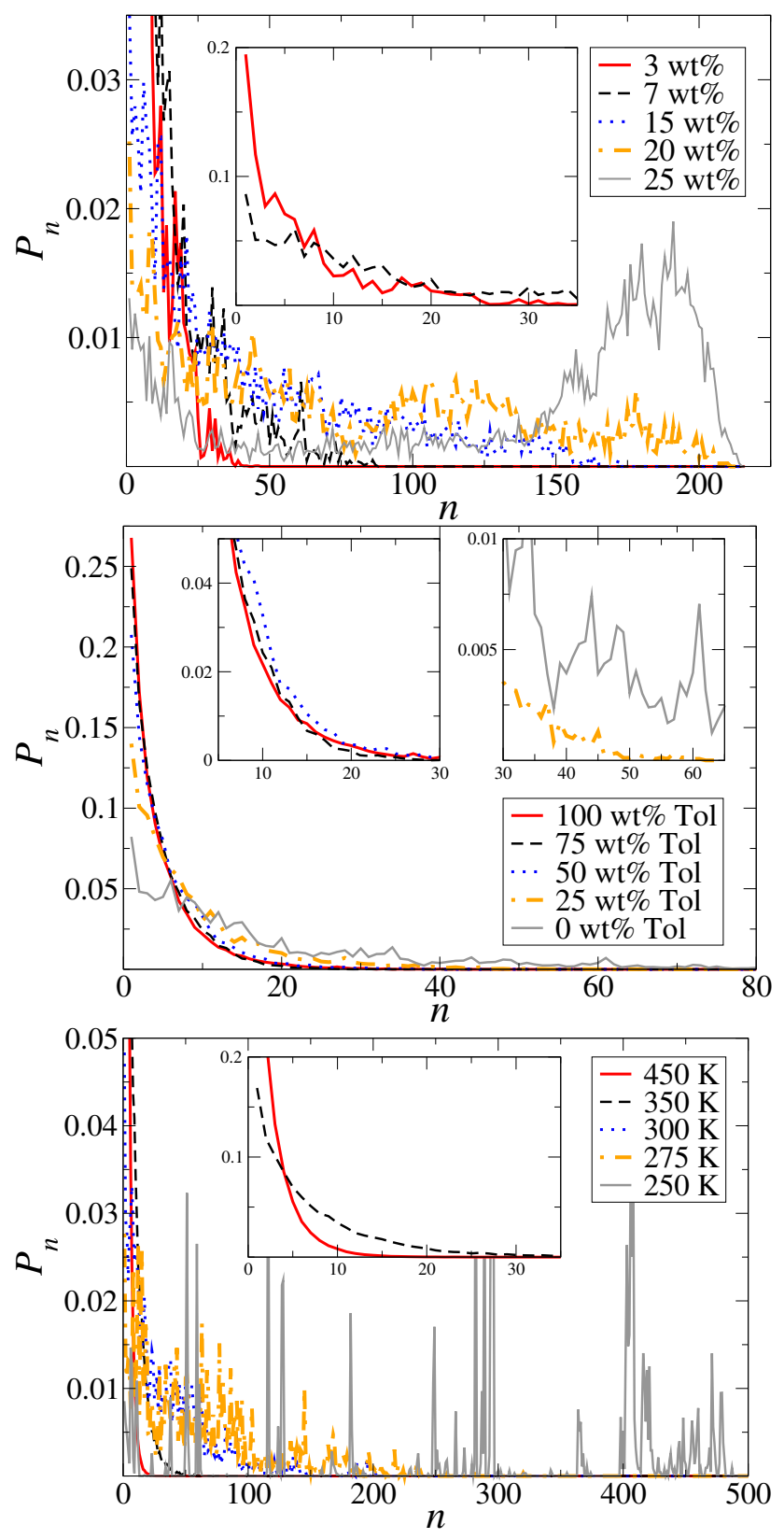

Figure 12: Cluster-size probabilities obtained from $(a)$ systems with 216 AsphC in explicit heptane at $\Phi_{\mathrm{Asph}}=3,7,15,20$, and $25 \mathrm{wt} \%$, simulated at $T=300 \mathrm{~K}$ and $P=1$ bar; $(b)$ systems with $216 \mathrm{AsphC}$ at $\Phi_{\mathrm{Asph}}=7 \mathrm{wt} \%$ in explicit mixtures of toluene and heptane at solvent compositions $x_{\text {tol }}=100,75,50,25$, and 0 (wt/wt $\%$ ), simulated at $T=300 \mathrm{~K}$ and $P=1$ bar; $(c)$ systems with 504 AsphC in explicit heptane at $\Phi_{\text {Asph }}=10 \mathrm{wt} \%$, simulated at $P=1$ bar, and $T=450,350300,275$, and $250 \mathrm{~K}$. 


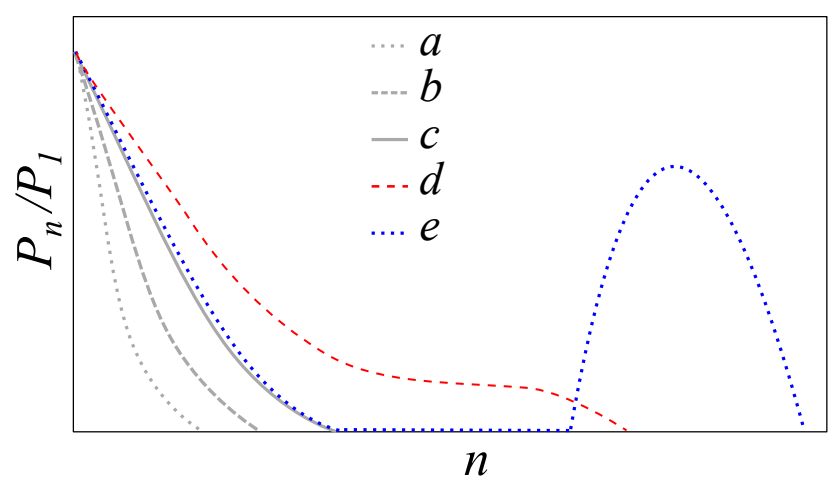

Figure 13: Schematic representation of cluster-size histograms seen in asphaltenic systems. Cases $(a),(b)$, and $(c)$ (gray) are identified as Type I, the curve $(d)$ (red) as Type II, and the histogram $(e)$ (blue) as Type III.

\section{Type I histograms: systems with low aggregation}

Systems with AsphC molecules in pure toluene at an asphaltene concentration of 7 wt\% and at a temperature of $300 \mathrm{~K}$ are selected as an example of low aggregation. System-size effects are tested using simulation boxes with 27 AsphC (from the validation tests, Fig. 8), 216 AsphC (from the solvent-composition series, Fig. 12(b)), and 504 AsphC molecules. The cluster-size probabilities are presented in Fig. 14, where small differences in the tails (see inset) are observed. It is clear that the 27 AsphC system does not exhibit structures with more than 20 molecules, presumably due to system size effects, while the other two systems have virtually the same behavior with structures of up to 30 molecules, being monitored. The difference in the tails between the smallest (27 AsphC) and the largest (504 AsphC) systems, considering the contributions from $n>12$, is in the order of one percent. When comparing the 216 and the 504 AsphC systems, the probability difference in the tails is just $0.2 \%$. Apparently, the system with 216 AsphC molecules is large enough to fully capture the aggregation details, but the results with the smallest system are still reliable at these conditions. The ensemble average cluster sizes are $\langle n\rangle=3.6 \pm 1.0,3.5 \pm 0.3$, and $3.5 \pm 0.2$, for the 27, 216, and 504 AsphC simulation boxes, respectively. Snapshots of equilibrium configurations are presented in Fig. 14, where disperse clusters of a few molecules are observed in the three systems, which in general are isotropic. To test for possible hysteresis, the 
temperature of the system with 216 AsphC asphaltenes is lowered to $260 \mathrm{~K}$ during $200 \mathrm{~ns}$, where a noticeable asphaltene aggregation is promoted, and then, raised back to $300 \mathrm{~K}$. The final equilibrium state is analyzed and the corresponding cluster-size distribution is included in Fig. 14, represented as a dotted blue line, which is seen to overlap the other histograms, indicating a clear reversibility of the equilibrium distributions.

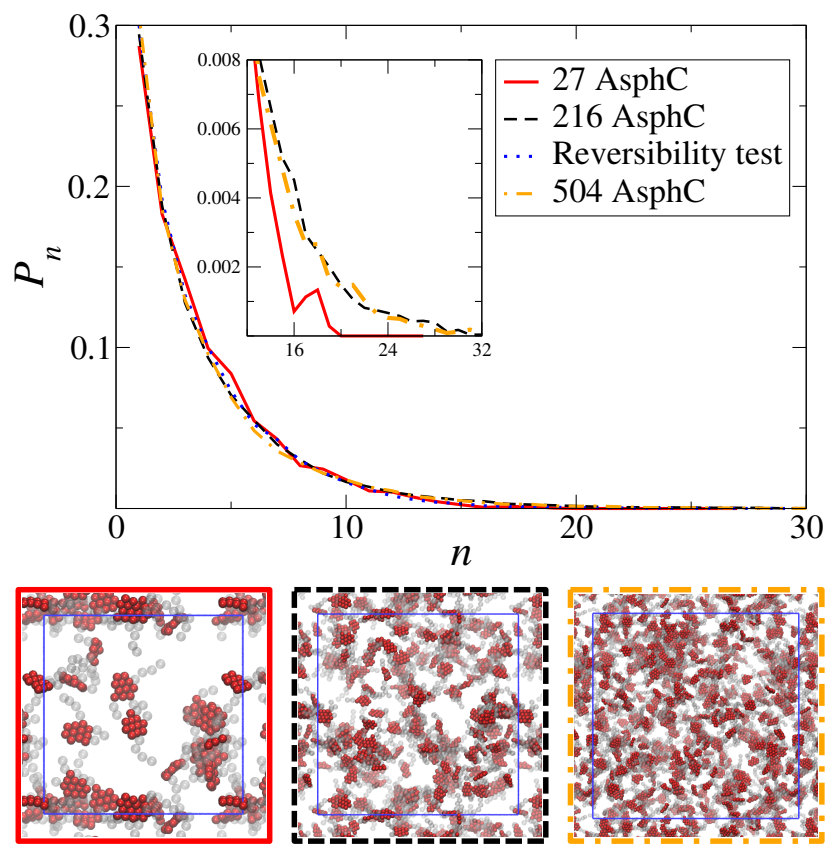

Figure 14: Cluster-size probabilities for systems of AsphC in toluene at $\Phi_{A s p h C}=7$ wt\%, $T=300 \mathrm{~K}$ and $P=1$ bar. The simulation boxes contain 27, 216, and 504 AsphC molecules. A reversibility test is run on the 216 AsphC system, from which the dotted histogram is obtained. The corresponding snapshots of equilibrium configurations are also shown.

To analyze the time dependence of this type of histograms, the maximum cluster size $n_{\max }$ as a function of time is plotted in Fig. 15. A small variation of $n_{\max }$ is observed along the equilibrium configurations in the last 200 ns of simulation, with values much smaller than the number of asphaltenes in the system, $n_{\max } \ll n_{\mathrm{Asph}}$. No time dependence is observed in the analysis of this system, which is consistent with the conclusions obtained for the analogous fully-atomistic simulations in Ref. 28. 


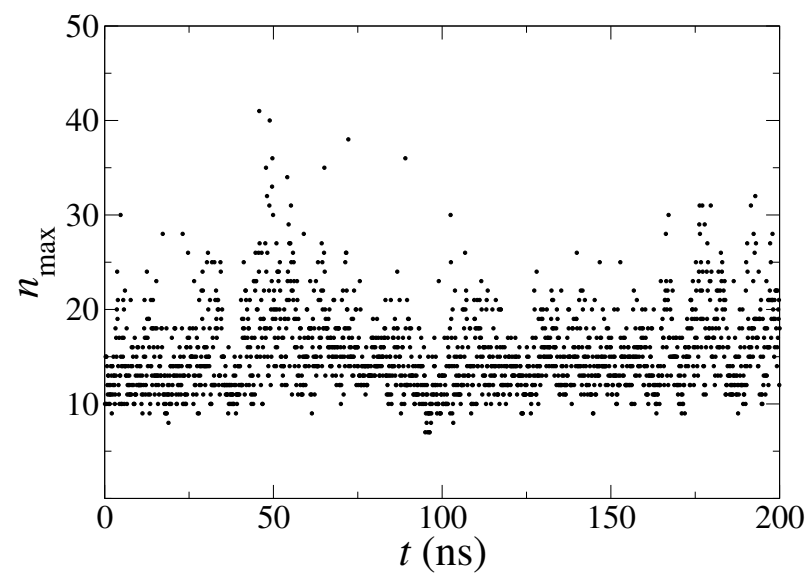

Figure 15: Maximum cluster-size value $n_{\max }$ as a function of time for the system with 504 AsphC in pure toluene at $\Phi_{\mathrm{Asph}}=7 \mathrm{wt} \%, T=300 \mathrm{~K}$, and $P=1$ bar.

\section{Type II histograms: intermediate aggregation}

As an example of systems with intermediate aggregation we choose the case of AsphC molecules in pure heptane at an asphaltene concentration of $20 \mathrm{wt} \%$, a temperature of 300 $\mathrm{K}$, and a pressure of 1 bar. System-size effects are tested using simulation boxes with 216 AsphC (from the asphaltene concentration series, Fig. 12(a)), 504 AsphC, 1000 AsphC, and 2000 AsphC molecules. The corresponding cluster-size probabilities are presented in Fig. 16, where data from ten histograms are fused into a single point to smooth the curves and ease visualization. A strong system-size dependence is evident in this case, with histogram shoulders spanning the full length of the graph for the system sizes for the 216, 504 and 1000 AsphC simulations. A second mode is observed in the distribution of the system with 2000 asphaltenes, which fits better with the histogram of a strongly aggregated system (defined here as Type III). Snapshots of some equilibrium configurations are also presented therein, where the two largest clusters of every system are highlighted in red and blue, and the rest of the molecules appear in grey. In general, interwoven thread-like structures are formed, which is consistent with simulations of similar CG asphaltene models in the literature ${ }^{55,81}$. The average cluster sizes are $\bar{n}=25 \pm 11,28 \pm 5,28 \pm 4$, and $31 \pm 3$, for the $216,504,1000$ and 2000 AsphC systems, respectively. These numerical averages are far from being representative of the physical picture provided by the histograms. The system-size dependence 
observed here is analogous to a simulation of a two-phase system, in which the "aggregate" grows as the system size is increased, forming actually the second phase ${ }^{108}$. To test if an energetic barrier could be preventing the full phase separation, a reversibility test is run over the system with 216 AsphC, by simulating it at a lower temperature, $T=260 \mathrm{~K}$, during $300 \mathrm{~ns}$ to promote large aggregates and then returning it to the original temperature of 300 $\mathrm{K}$. The equilibrium state is analyzed and the resulting cluster-size histogram is included in Fig. 16. The distributions from both 216 AsphC systems (the one with an initially random configuration and the one with an initial two-phase configuration), are practically the same, and hence no reversibility effects are observed in this test.
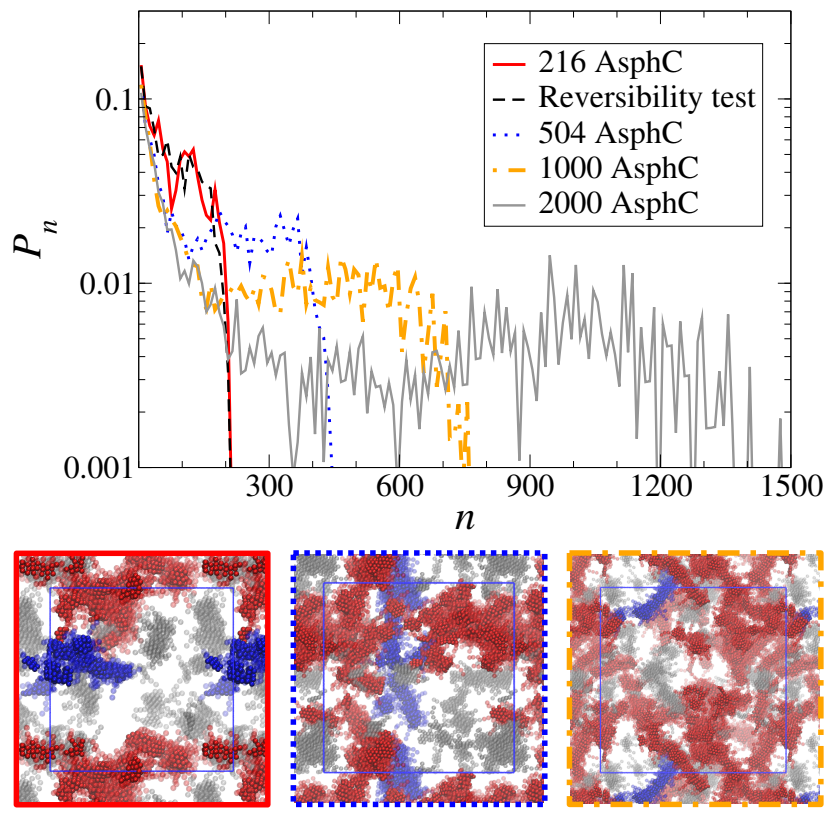

Figure 16: Smoothed Cluster-size probabilities for systems of AsphC in heptane at $\Phi_{\text {AsphC }}=$ $20 \mathrm{wt} \%, T=300 \mathrm{~K}$, and $P=1$ bar. The simulation boxes contain 216, 504, 1000, and 2000 AsphC molecules. A reversibility test is run over the 216 AsphC system, from which the dashed black histogram is obtained. The snapshots of equilibrium configurations are shown for the systems with 216, 504, and 1000 asphaltenes, where the red molecules are part of the largest cluster in the system, the blue molecules are part of the second largest cluster, and the remaining molecules are shown in grey.

To elucidate time-dependence features of this type of systems, data from the 1000 AsphC system are used to calculate the maximum cluster size $n_{\max }$ along the equilibrium trajectory. In Fig. $17(a), n_{\max }$ as a function of time is plotted, showing a large variation of this quantity, 
with some configurations where the largest cluster contains 200 asphaltenes and other frames where the largest cluster contains more than 600 molecules. The details of the "instantaneous" histograms are further obtained by separating the configurations into two groups: those with $n_{\max } \leq 450$ and those with $n_{\max }>450$, with the aim of gathering alike simulation frames into each group and capture the representative aggregation features for each case. The division is indicated with a line in Fig. 17(a). The smoothed histograms from both groups are presented in Fig. 17(b), including the histogram obtained from the overall analysis. An interpretation based on the numerical detail is not appropriate since the selection of the cutoff value $n_{\text {cut }}=450$ is arbitrary, however, an important message is extracted from this plot: the initially reported cluster-size distribution is really a superposition of two instantaneous states. In one state, a large "aggregate" (asphaltene-rich phase) coexists with smaller supernatant aggregates (solvent-rich phase), having a range of $n$ values that are not accessible in those configurations. In the other state, histogram $n_{\max } \leq 450$, the distribution is continuous and the region that seemed forbidden in the previous case is here populated, consequence of the instantaneous breaking of a very large structure. The visualization of a large cluster and its further breaking into two structures is shown in Fig. 17(c). The cluster-size averages are $\bar{n}=27$ and 29 from the partial histograms, $n_{\max } \leq 450$ and $n_{\max }>450$, respectively. Then, the numerical averages are very similar to the overall value $\bar{n}=28$, despite the notable differences in the distributions. The cluster-size range accessible in the breaking and recombination of the clusters must be related to the system size, which, connected with the previous test, points to Type II histograms as phase-separated systems with artifacts due to the finite system size.

\section{Type III histograms: systems with strong aggregation}

To understand the features of systems with strong aggregation, simulation boxes of AsphC molecules in pure heptane are studied at an asphaltene concentration of $25 \mathrm{wt} \%$, at $300 \mathrm{~K}$ and 1 bar. System-size effects are examined by simulating boxes with 216, 504 (from the 

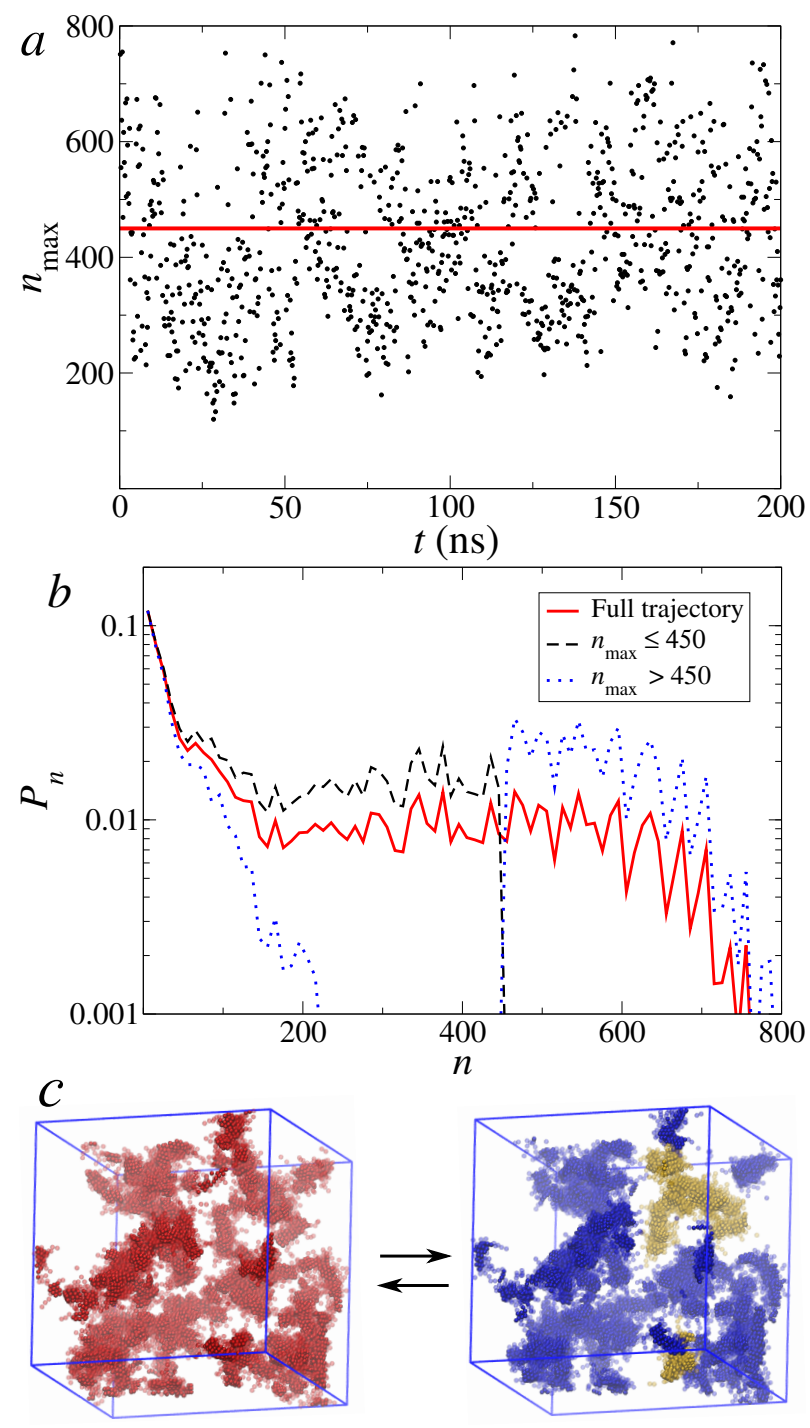

Figure 17: (a) Maximum cluster-size value $n_{\max }$ as a function of time, for the system with 1000 AsphC in pure heptane at $\Phi_{\mathrm{Asph}}=20 \mathrm{wt} \%, T=300 \mathrm{~K}$, and $P=1$ bar. An arbitrary cutoff value $n_{\text {cut }}=450$ is indicated with a horizontal line. (b) Cluster-size probabilities from the analysis of all the configurations and partial cluster-size distributions obtained from the analysis of simulation frames with $n_{\text {max }} \leq n_{\text {cut }}$ or from the analysis of frames with $n_{\max }>n_{\text {cut }}$. These histograms are smoothed versions of the original output to ease visualization, every point represents ten histograms that were merged. (c) Visualization of one large cluster identified in the simulation box and its breaking into two smaller structures. 
asphaltene concentration series, Fig. 12(a)), and 1000 AsphC. In Fig. 18, a smoothed version (ten histograms are fused into one point to ease visualization) of the resulting cluster-size probabilities are shown with the corresponding equilibrium snapshots, where the largest structure is colored in red, the second largest cluster in blue, and the remaining molecules are represented in grey. In this case, the underlying columnar arrangement of molecules becomes more distorted as the different threads fuse by T-shaped or offset-parallel stacking. The average cluster sizes are $\bar{n}=39 \pm 16,53 \pm 16$, and $54 \pm 13$, for the 216,504 , and 1000 AsphC systems, respectively. By comparing the averages, it seems that the system with 504 AsphC molecules reaches a representative value, however, the cluster-size distributions exhibit a strong system-size dependence. This dependence is consistent with the simulation of a system under phase-separation, where the size of the large "aggregate" increases with the system size as it is representative of a macrophase. In a gedankenexperiment, in which we continue increasing the simulation box up to macroscopic dimensions, we would expect to observe an asphaltene-rich phase in equilibrium with a solvent-rich phase. The limiting cluster-size distribution would have a size-independent Type I section (related to the solvent rich phase, in which a distribution of monomers, dimers, and relatively small clusters would be observed) and a second peak that would continue moving as the system size is increased. The conserved quantity in this ensemble is the composition of both phases.

The time evolution of this type of histograms is analyzed using the simulation box with 1000 AsphC. The maximum cluster size $n_{\max }$ through the trajectory is presented in Fig. 19(a). A large variation of $n_{\max }$ is observed, which is related to the breaking of large clusters alternated with the merging of the smaller structures into large aggregates. Some frames present aggregates as large as 900 asphaltenes. The "instantaneous" histograms are obtained by separating the simulation frames into two groups: those with $n_{\max } \leq 650$ and those with $n_{\max }>650$, the separation is represented as a dashed line in Fig. 19(a). The smoothed versions of the histograms obtained from both groups are presented in Fig. 19(b). We observe that the cluster-size distribution represents the superposition of configurations with very 

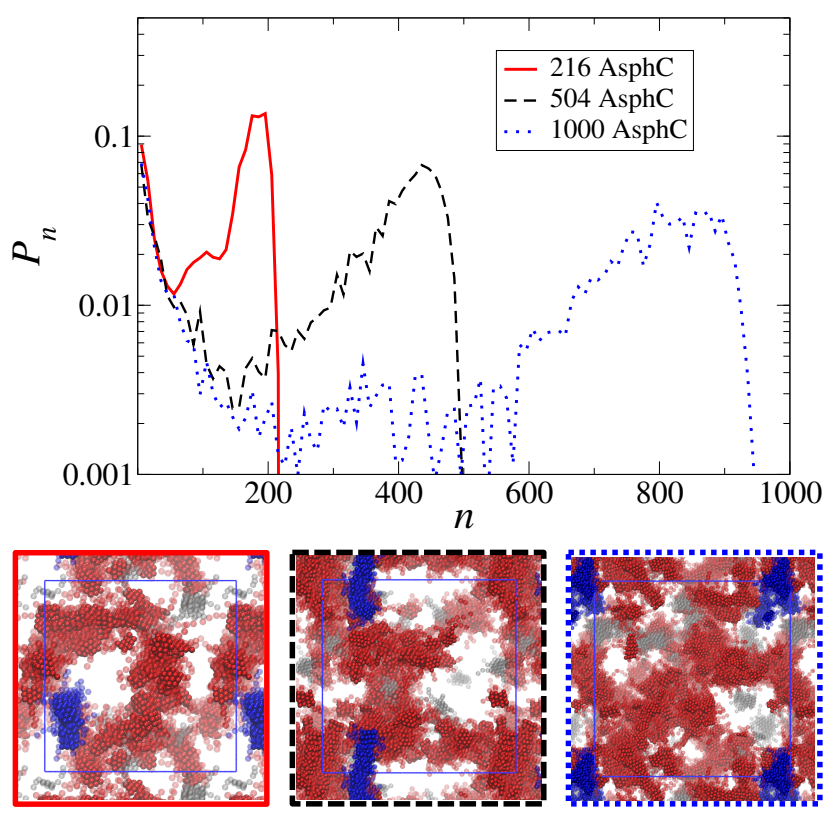

Figure 18: Cluster-size probabilities for systems of AsphC in pure heptane at $\Phi_{\text {AsphC }}=25$ wt $\%, T=300 \mathrm{~K}$, and $P=1$ bar. The simulation boxes contain 216, 504, and 1000 AsphC molecules. The corresponding snapshots of equilibrium configurations are also shown, where the red molecules are part of the largest cluster in the system, the blue molecules are part of the second largest cluster, and the remaining molecules are shown in grey.

large clusters, coexisting with smaller structures, and instantaneous configurations where the large clusters are broken, with a predominance of the first state in this example.

\section{Conclusions}

We present a systematic procedure to determine Mie potential force fields for PAHs with arbitrary shapes, such as those encountered within the aromatic cores of asphaltenic molecular structures. These coarse-grained moieties are combined in a group-contribution fashion using CG force-fields for aliphatic molecules obtained through the SAFT- $\gamma$ Mie EoS ${ }^{52,84,94}$ to provide for models of a continental-type and an archipelago-type asphaltene ${ }^{28,29}$. The development of the model involved the use of information from fully atomistic simulations to tune flexibility and unlike-interaction parameters, which we suspect may be transferred into other models. We note, however, that both features have a strong effect on the molecular arrangement of molecules within a cluster and thus drive the size and shape of the supramolecular 

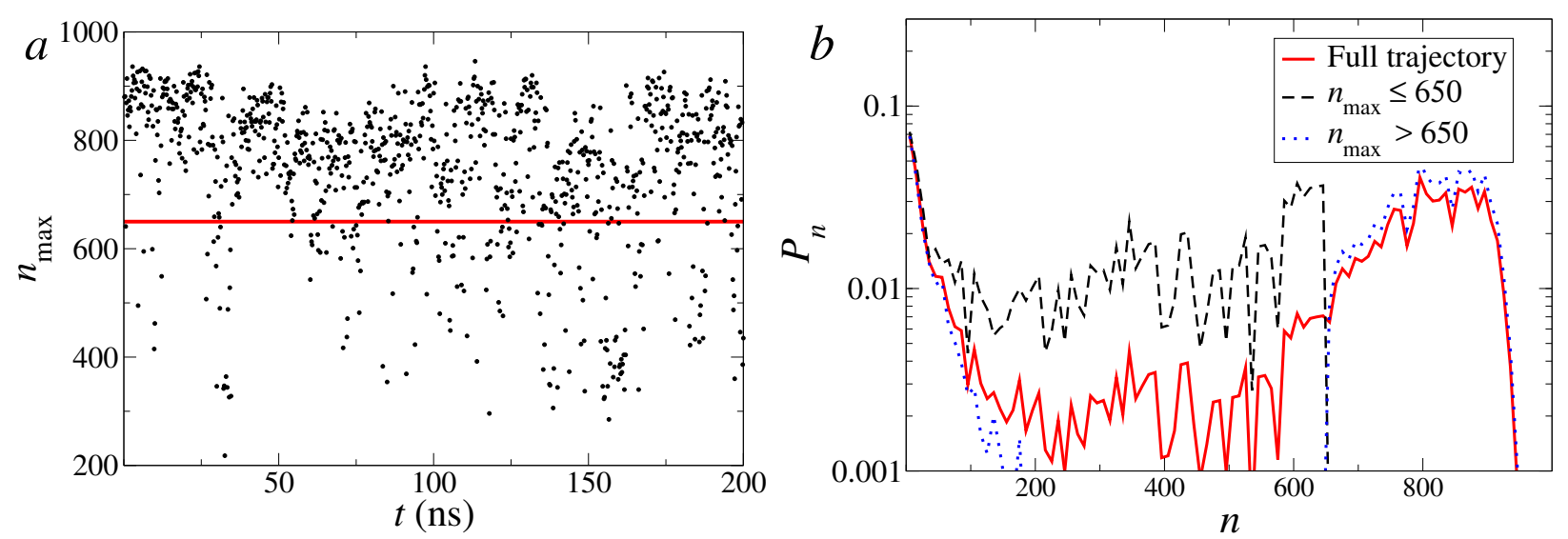

Figure 19: (a) Maximum cluster-size value $n_{\max }$ as a function of time, for the system with 1000 AsphC in pure heptane at $\Phi_{\mathrm{Asph}}=25 \mathrm{wt} \%, T=300 \mathrm{~K}$, and $P=1$ bar. An arbitrary cutoff value $n_{\text {cut }}=650$ is indicated with a horizontal line. (b) Cluster-size probabilities from the analysis of all the configurations and partial cluster-size distributions obtained from the analysis of simulation frames with $n_{\text {max }} \leq n_{\text {cut }}$ or from the analysis of frames with $n_{\max }>n_{\text {cut }}$. These histograms are smoothed versions of the original output, and every point is representing ten histograms that were merged.

structures. The aggregation properties of the final CG asphaltene systems are compared with fully-atomistic simulations from the literature ${ }^{28}$, finding an excellent qualitative and quantitative agreement between both levels of description for structural properties of clusters. The proposed procedure is simple and reliable enough to produce the CG parameters of asphaltenic molecules, however, further modeling of other structures is needed to assess the generality of the methodology and its limitations. Also, we stress that fundamental research is necessary to obtain CG building blocks for arbitrary PAHs from first principles.

In the second part of this work, the CG AsphC model is exploited in large-scale MD simulations with up to 2000 asphaltenes in explicit solvent to study the aggregation behavior at different conditions, using cluster-size distributions as the main aggregation indicator. The trends in the simulations are in qualitative agreement with the literature observations $^{8,16,17,21,30,75,81}$ : the aggregation is promoted as the asphaltene concentration is increased, as the amount of heptane in the solvent is increased, or as the temperature is decreased. A closer inspection of some selected cases, suggests that the cluster-size distribution should be averaged over a large group of equilibrium configurations to include as much 
information as possible, but that such an average curve should be carefully interpreted. We note that the information contained in the distributions should not be reduced to the average cluster size unless there is certainty that no system-size effects are present, e.g. the systems we called Type I in our categorization. The shape and details of the Type I histogram must be dependent of the thermodynamic conditions and molecular structure, but the main feature of that category is the lack of system-size dependence and lack of hysteresis, which are indications of good solubility conditions. The asymmetric nature of the mixture makes this distribution of $n$-mers more evident, but there is no evidence of any type of preferential cluster or nanoaggregate of consistent and reproducible size. Upon changes in thermodynamic conditions towards unfavorable solubility, the systems present a phase split consistent with the physical description provided by Sirota ${ }^{8,16}$. In the tests performed, the intermediate histograms (Type II) were observed to be phase-separated systems (Type III) stabilized by simulation boxes which are too small. However, we do not discard metastability as the cause of such behavior in other cases where slow kinetics are involved in the simulations as well as in the real systems ${ }^{17}$. The study of polydisperse asphaltenic systems would also provide further understanding of the aggregation process and properties, as discussed for example in Refs. $24,30,109$.

\section{Supporting information}

Comments on benzene as a building block of CG PAHs. Tests of the proposed CG PAHs. Asphaltene C topology. Asphaltene A topology. List of simulation systems. Cluster properties.

\section{Acknowledgments}

The authors would like to acknowledge the funding and technical support from BP through the BP International Centre for Advanced Materials (BP-ICAM) which made this research 
possible. Part of this work was supported by the EPSRC through research grants to the Molecular Systems Engineering group (Grants EP/EP016340 and EP/J014958). The simulations described herein were performed using the facilities of the Imperial College High Performance Computing Service and the BP High Performance Computer Centre.

\section{References}

(1) Ovalles, C., Moir, M. E., Eds. The Boduszynski Continuum: Contributions to the Understanding of the Molecular Composition of Petroleum; ACS Symposim Series: Washington, 2018; Vol. 1282.

(2) Mullins, O. C.; Sheu, E. Y.; Hammami, A.; Marshall, A. G. Asphaltenes, Heavy Oils, and Petroleomics; Springer Science \& Business Media: New York, 2007.

(3) Artola, P.-A.; Pereira, F.; Adjiman, C.; Galindo, A.; Müller, E. A.; Jackson, G.; Haslam, A. Understanding the Fluid Phase Behaviour of Crude Oil: Asphaltene Precipitation. Fluid Phase Equilib. 2011, 306, 129-136.

(4) Pedersen, K.; Christensen, P.; Shaikh, J. Phase Behavior of Petroleum Reservoir Fluids; CRC Press: Boca Raton, 2012.

(5) Wiehe, I. Asphaltene Solubility and Fluid Compatibility. Energy Fuels 2012, 26, 40044016.

(6) Coletti, F.; Hewitt, G. Crude Oil Fouling: Deposit Characterization, Measurements, and Modeling; Gulf Professional Publishing, 2014.

(7) Greenfield, M. Molecular Modelling and Simulation of Asphaltenes and Bituminous Materials. Int. J. Pavement Eng. 2011, 12, 325-341.

(8) Sirota, E. Physical Structure of Asphaltenes. Energy Fuels 2005, 19, 1290-1296. 
(9) Pinkston, D. S.; Duan, P.; Gallardo, V. A.; Habicht, S. C.; Tan, X.; Qian, K.; Gray, M.; Mullen, K.; Kenttamaa, H. I. Analysis of Asphaltenes and Asphaltene Model Compounds by Laser-Induced Acoustic Desorption/Fourier Transform Ion Cyclotron Resonance Mass Spectrometry. Energy Fuels 2009, 23, 5564-5570.

(10) Sheremata, J. M.; Gray, M. R.; Dettman, H. D.; McCaffrey, W. C. Quantitative Molecular Representation and Sequential Optimization of Athabasca Asphaltenes. Energy Fuels 2004, 18, 1377-1384.

(11) Boek, E. S.; Yakovlev, D. S.; Headen, T. F. Quantitative Molecular Representation of Asphaltenes and Molecular Dynamics Simulation of their Aggregation. Energy Fuels 2009, 23, 1209-1219.

(12) Martín-Martínez, F. J.; Fini, E. H.; Buehler, M. J. Molecular Asphaltene Models Based on Clar Sextet Theory. RSC Advances 2015, 5, 753-759.

(13) Alvarez-Ramírez, F.; Ruiz-Morales, Y. Island versus Archipelago Architecture for Asphaltenes: Polycyclic Aromatic Hydrocarbon Dimer Theoretical Studies. Energy Fuels 2013, 2\%, 1791-1808.

(14) Sabbah, H.; Morrow, A. L.; Pomerantz, A. E.; Zare, R. N. Evidence for Island Structures as the Dominant Architecture of Asphaltenes. Energy Fuels 2011, 25, 1597-1604.

(15) Mullins, O. C.; Sabbah, H.; Eyssautier, J.; Pomerantz, A. E.; Barré, L.; Andrews, A. B.; Ruiz-Morales, Y.; Mostowfi, F.; McFarlane, R.; Goual, L., et al. Advances in Asphaltene Science and the Yen-Mullins Model. Energy Fuels 2012, 26, 3986-4003.

(16) Sirota, E. B.; Lin, M. Y. Physical Behavior of Asphaltenes. Energy Fuels 2007, 21, 2809-2815.

(17) Fávero, C. V. B.; Maqbool, T.; Hoepfner, M.; Haji-Akbari, N.; Fogler, H. S. Revisiting 
the Flocculation Kinetics of Destabilized Asphaltenes. Adv. Colloid. Interface Sci. 2017, 244, 267-280.

(18) Haji-Akbari, N.; Masirisuk, P.; Hoepfner, M. P.; Fogler, H. S. A Unified Model for Aggregation of Asphaltenes. Energy Fuels 2013, 27, 2497-2505.

(19) Adams, J. J. Asphaltene Adsorption, a Literature Review. Energy Fuels 2014, 28, $2831-2856$.

(20) Sjöblom, J.; Simon, S.; Xu, Z. Model Molecules Mimicking Asphaltenes. Adv. Colloid Interface Sci. 2015, 218, 1-16.

(21) Gray, M. R.; Tykwinski, R. R.; Stryker, J. M.; Tan, X. Supramolecular Assembly Model for Aggregation of Petroleum Asphaltenes. Energy Fuels 2011, 25, 3125-3134.

(22) Murgich, J.; Rodríguez, J.; Aray, Y. Molecular Recognition and Molecular Mechanics of Micelles of Some Model Asphaltenes and Resins. Energy Fuels 1996, 10, 68-76.

(23) Schuler, B.; Meyer, G.; Peña, D.; Mullins, O. C.; Gross, L. Unraveling the Molecular Structures of Asphaltenes by Atomic Force Microscopy. JACS 2015, 137, 9870-9876.

(24) Acevedo, S.; Castillo, J.; Vargas, V.; Castro, A.; Delgado, O. Z.; Ariza, C. A. F.; Cortés, F. B.; Bouyssiere, B. Suppression of Phase Separation as a Hypothesis to Account for Nuclei or Nanoaggregate Formation by Asphaltenes in Toluene. Energy Fuels 2018, 32, 6669-6677.

(25) Subramanian, S.; Simon, S.; Sjöblom, J. Asphaltene Precipitation Models: a Review. J. Dispersion Sci. Technol. 2016, 37, 1027-1049.

(26) Mullins, O. C.; Pomerantz, A. E.; Zuo, J. Y.; Dong, C. Downhole Fluid Analysis and Asphaltene Science for Petroleum Reservoir Evaluation. Annu. Rev. Chem. Biomol. Eng. 2014, 5, 325-345. 
(27) Forte, E.; Taylor, S. E. Thermodynamic Modelling of Asphaltene Precipitation and Related Phenomena. Adv. Colloid Interface Sci. 2015, 217, 1-12.

(28) Headen, T. F.; Boek, E. S.; Jackson, G.; Totton, T. S.; Müller, E. A. Simulation of Asphaltene Aggregation through Molecular Dynamics: Insights and Limitations. Energy Fuels 2017, 31, 1108-1125.

(29) Headen, T. F.; Boek, E. S.; Skipper, N. T. Evidence for Asphaltene Nanoaggregation in Toluene and Heptane from Molecular Dynamics Simulations. Energy Fuels 2009, 23, 1220-1229.

(30) Javanbakht, G.; Sedghi, M.; Welch, W. R.; Goual, L.; Hoepfner, M. P. Molecular Polydispersity Improves Prediction of Asphaltene Aggregation. J. Mol. Liq. 2018, 256, 382-394.

(31) Ungerer, P.; Rigby, D.; Leblanc, B.; Yiannourakou, M. Sensitivity of the Aggregation Behaviour of Asphaltenes to Molecular Weight and Structure Using Molecular Dynamics. Mol. Simul. 2014, 40, 115-122.

(32) Silva, H. S.; Sodero, A. C.; Bouyssiere, B.; Carrier, H.; Korb, J.-P.; Alfarra, A.; Vallverdu, G.; Bégué, D.; Baraille, I. Molecular Dynamics Study of Nanoaggregation in Asphaltene Mixtures: Effects of the N, O, and S Heteroatoms. Energy Fuels 2016, 30, 5656-5664.

(33) Sodero, A. C.; Santos Silva, H.; Guevara Level, P.; Bouyssiere, B.; Korb, J.-P.; Carrier, H.; Alfarra, A.; Begue, D.; Baraille, I. Investigation of the Effect of Sulfur Heteroatom on Asphaltene Aggregation. Energy Fuels 2016, 30, 4758-4766.

(34) Sedghi, M.; Goual, L.; Welch, W.; Kubelka, J. Effect of Asphaltene Structure on Association and Aggregation Using Molecular Dynamics. J. Phys. Chem. B 2013, $117,5765-5776$. 
(35) Zhu, X.; Wu, G.; Coulon, F.; Wu, L.; Chen, D. Correlating Asphaltene Dimerization with its Molecular Structure by Potential of Mean Force Calculation and Data Mining. Energy Fuels 2018, 32, 5779-5788.

(36) Pacheco-Sánchez, J.; Zaragoza, I.; Martínez-Magadán, J. Asphaltene Aggregation under Vacuum at Different Temperatures by Molecular Dynamics. Energy Fuels 2003, $17,1346-1355$.

(37) Li, D. D.; Greenfield, M. L. Viscosity, Relaxation Time, and Dynamics within a Model Asphalt of Larger Molecules. J. Chem. Phys. 2014, 140, 034507.

(38) Zhang, L.; Greenfield, M. L. Rotational Relaxation Times of Individual Compounds Within Simulations of Molecular Asphalt Models. J. Chem. Phys. 2010, 132, 184502.

(39) Kuznicki, T.; Masliyah, J. H.; Bhattacharjee, S. Molecular Dynamics Study of Model Molecules Resembling Asphaltene-Like Structures in Aqueous Organic Solvent Systems. Energy Fuels 2008, 22, 2379-2389.

(40) Murgich, J. Molecular Simulation and the Aggregation of the Heavy Fractions in Crude Oils. Mol. Simul. 2003, 29, 451-461.

(41) Rogel, E.; León, O. Study of the Adsorption of Alkyl-Benzene-Derived Amphiphiles on an Asphaltene Surface Using Molecular Dynamics Simulations. Energy Fuels 2001, 15, 1077-1086.

(42) Gavrilenko, L.; Klauda, J. B. Aggregation of Modified Hexabenzocoronenes as Models for Early Stage Asphaltene Self-Assembly. Mol. Simul. 2018, 1-12.

(43) Jover, J. F.; Müller, E. A.; Haslam, A. J.; Galindo, A.; Jackson, G.; Toulhoat, H.; Nieto-Draghi, C. Aspects of Asphaltene Aggregation Obtained from Coarse-Grained Molecular Modeling. Energy Fuels 2015, 29, 556-566. 
(44) von Lilienfeld, O. A.; Andrienko, D. Coarse-Grained Interaction Potentials for Polyaromatic Hydrocarbons. J. Chem. Phys. 2006, 124, 054307.

(45) Soper, A. Empirical Potential Monte Carlo Simulation of Fluid Structure. Chem. Phys. 1996, 202, 295-306.

(46) Müller-Plathe, F. Coarse-Graining in Polymer Simulation: from the Atomistic to the Mesoscopic Scale and Back. ChemPhysChem 2002, 3, 754-769.

(47) Chu, J.-W.; Ayton, G.; Izvekov, S.; Voth, G. Emerging Methods for Multiscale Simulation of Biomolecular Systems. Mol. Phys. 2007, 105, 167-175.

(48) Shell, M. S. In Advances in Chemical Physics; Rice, S. A., Dinner, A. R., Eds.; John Wiley \& Sons, Inc.: Hoboken, NJ, USA, 2016; Vol. 161.

(49) Müller, E. A.; Jackson, G. Force-Field Parameters from the SAFT- $\gamma$ Equation of State for Use in Coarse-Grained Molecular Simulations. Annu. Rev. Chem. Biomol. Eng. 2014, 5, 405-427.

(50) Papaioannou, V.; Lafitte, T.; Avendaño, C.; Adjiman, C. S.; Jackson, G.; Müller, E. A.; Galindo, A. Group Contribution Methodology Based on the Statistical Associating Fluid Theory for Heteronuclear Molecules Formed from Mie Segments. J. Chem. Phys. 2014, 140, 054107.

(51) Avendaño, C.; Lafitte, T.; Adjiman, C. S.; Galindo, A.; Müller, E. A.; Jackson, G. SAFT- $\gamma$ Force Field for the Simulation of Molecular Fluids: 2. Coarse-Grained Models of Greenhouse Gases, Refrigerants, and Long Alkanes. J. Phys. Chem. B 2013, 117, $2717-2733$.

(52) Mejía, A.; Herdes, C.; Müller, E. A. Force Fields for Coarse-Grained Molecular Simulations from a Corresponding States Correlation. Ind. Eng. Chem. Res. 2014, 53, 4131-4141. 
(53) Marrink, S. J.; Risselada, H. J.; Yefimov, S.; Tieleman, D. P.; De Vries, A. H. The MARTINI Force Field: Coarse Grained Model for Biomolecular Simulations. J. Phys. Chem. B 2007, 111, 7812-7824.

(54) Marrink, S. J.; Tieleman, D. P. Perspective on the Martini Model. Chem. Soc. Rev. 2013, 42, 6801-6822.

(55) Wang, J.; Ferguson, A. L. Mesoscale Simulation of Asphaltene Aggregation. J. Phys. Chem. B 2016, 120, 8016-8035.

(56) Hsu, D. D.; Xia, W.; Arturo, S. G.; Keten, S. Thermomechanically Consistent and Temperature Transferable Coarse-Graining of Atactic Polystyrene. Macromolecules 2015, 48, 3057-3068.

(57) Rahman, S.; Lobanova, O.; Jiménez-Serratos, G.; Braga, C.; Raptis, V.; Muller, E. A.; Jackson, G.; Avendano, C.; Galindo, A. SAFT- $\gamma$ Force Field for the Simulation of Molecular Fluids. 5. Hetero-Group Coarse-Grained Models of Linear Alkanes and the Importance of Intramolecular Interactions. J. Phys. Chem. B 2018, 122, 9161-9177.

(58) Mansbach, R. A.; Ferguson, A. L. Coarse-Grained Molecular Simulation of the Hierarchical Self-Assembly of $\pi$-Conjugated Optoelectronic Peptides. J. Phys. Chem. B 2017, 121, 1684-1706.

(59) Carbone, P.; Varzaneh, H. A. K.; Chen, X.; Müller-Plathe, F. Transferability of Coarse-Grained Force Fields: The Polymer Case. J. Chem. Phys. 2008, 128, 064904.

(60) Foley, T. T.; Shell, M. S.; Noid, W. The Impact of Resolution upon Entropy and Information in Coarse-Grained Models. J. Chem. Phys. 2015, 143, 12B601_1.

(61) Brini, E.; Algaer, E. A.; Ganguly, P.; Li, C.; Rodríguez-Ropero, F.; van der Vegt, N. F. Systematic Coarse-Graining Methods for Soft Matter Simulations-a Review. Soft Matter 2013, 9, 2108-2119. 
(62) Potter, T. D.; Tasche, J.; Wilson, M. R. Assessing the Transferability of Common Top-Down and Bottom-Up Coarse-Grained Molecular Models for Molecular Mixtures. Phys. Chem. Chem. Phys. 2019, 21, 1912-1927.

(63) Müller, E. A.; Mejía, A. Extension of the SAFT-VR Mie EoS to Model Homonuclear Rings and its Parametrization Based on the Principle of Corresponding States. Langmuir 2017, 33, 11518-11529.

(64) Heinemann, T.; Palczynski, K.; Dzubiella, J.; Klapp, S. H. Coarse-Grained Electrostatic Interactions of Coronene: Towards the Crystalline Phase. J. Chem. Phys. 2015, $143,174110$.

(65) Srinivas, G.; Klein, M. L. Molecular Dynamics Simulations of Self-Assembly and Nanotube Formation by Amphiphilic Molecules in Aqueous Solution: a Coarse-Grain Approach. Nanotechnology 2007, 18, 205703.

(66) Hernández-Rojas, J.; Calvo, F.; Niblett, S.; Wales, D. Dynamics and Thermodynamics of the Coronene Octamer Described by Coarse-Grained Potentials. Phys. Chem. Chem. Phys. 2017, 19, 1884-1895.

(67) Potter, T. D.; Tasche, J.; Barrett, E. L.; Walker, M.; Wilson, M. R. Development of New Coarse-Grained Models for Chromonic Liquid Crystals: Insights from Top-Down Approaches. Liquid Crystals 2017, 44, 1979-1989.

(68) Tripathy, M.; Agarwal, U.; Kumar, P. S. Toward Transferable Coarse-Grained Potentials for Poly-Aromatic Hydrocarbons: A Force Matching Approach. Macromol. Theory Simul. 2018, 1800040.

(69) Rapacioli, M.; Spiegelman, F.; Talbi, D.; Mineva, T.; Goursot, A.; Heine, T.; Seifert, G. Correction for Dispersion and Coulombic Interactions in Molecular Clusters with Density Functional Derived Methods: Application to Polycyclic Aromatic Hydrocarbon Clusters. J. Chem. Phys. 2009, 130, 244304. 
(70) Castellano, O.; Gimon, R.; Soscun, H. Theoretical Study of the $\sigma-\pi$ and $\pi-\pi$ Interactions in Heteroaromatic Monocyclic Molecular Complexes of Benzene, Pyridine, and Thiophene Dimers: Implications on the Resin-Asphaltene Stability in Crude Oil. Energy Fuels 2011, 25, 2526-2541.

(71) Ruiz-Morales, Y. The Agreement Between Clar Structures and Nucleus-Independent Chemical Shift Values in Pericondensed Benzenoid Polycyclic Aromatic Hydrocarbons: an Application of the Y-Rule. J. Phys. Chem. A 2004, 108, 10873-10896.

(72) Rury, A. S.; Ferry, C.; Hunt, J. R.; Lee, M.; Mondal, D.; O’Connell, S. M.; Phan, E. N.; Peng, Z.; Pokhilko, P.; Sylvinson, D., et al. Solvent Thermodynamic Driving Force Controls Stacking Interactions Between Polyaromatics. J. Phys. Chem. C 2016, 120, $23858-23869$.

(73) Yiannourakou, M.; Ungerer, P.; Lachet, V.; Rousseau, B.; Teuler, J.-M. United Atom Forcefield for Vapor-Liquid Equilibrium (VLE) Properties of Cyclic and Polycyclic Compounds from Monte Carlo Simulations. Fluid Phase Equilib. 2019, 481, 28-43.

(74) Kim, S.-H.; Kim, K.-D.; Lee, H.; Lee, Y.-K. Beneficial Roles of H-donors as Diluent and H-shuttle for Asphaltenes in Catalytic Upgrading of Vacuum Residue. Chem. Eng. J. 2017, 314, 1-10.

(75) Wang, J.; Gayatri, M. A.; Ferguson, A. L. Mesoscale Simulation and Machine Learning of Asphaltene Aggregation Phase Behavior and Molecular Assembly Landscapes. J. Phys. Chem. B 2017, 121, 4923-4944.

(76) Ervik, A.; Lysgaard, M. O.; Herdes, C.; Jiménez-Serratos, G.; Müller, E. A.; Munkejord, S. T.; Müller, B. A Multiscale Method for Simulating Fluid Interfaces Covered with Large Molecules such as Asphaltenes. J. Comput. Phys. 2016, 327, 576-611.

(77) Zhang, S.-F.; Sun, L.-L.; Xu, J.-B.; Wu, H.; Wen, H. Aggregate Structure in Heavy 
Crude Oil: Using a Dissipative Particle Dynamics Based Mesoscale Platform. Energy Fuels 2010, 24, 4312-4326.

(78) Aguilera-Mercado, B.; Herdes, C.; Murgich, J.; Müller, E. A. Mesoscopic Simulation of Aggregation of Asphaltene and Resin Molecules in Crude Oils. Energy Fuels 2006, 20, 327-338.

(79) Desgranges, C.; Delhommelle, J. Coarse-Grained Model and Boiling Point Prediction for Asphaltene Model Compounds via HMC-WL Simulations. Energy \& Fuels 2017, 31, 10699-10705.

(80) Wang, J.; Gayatri, M.; Ferguson, A. L. Coarse-Grained Molecular Simulation and Nonlinear Manifold Learning of Archipelago Asphaltene Aggregation and Folding. $J$. Phys. Chem. B 2018,

(81) Lee, H.; Lee, Y.-K. Effects of the Asphaltene Structure and the Tetralin/Heptane Solvent Ratio on the Size and Shape of Asphaltene Aggregates. Phys. Chem. Chem. Phys. 2017, 19, 13931-13940.

(82) Chen, J.; Chen, J.; Zhong, C.; Chen, S.; Chen, B.; Fang, S.; Xiang, W. Mesoscopic Probes in Asphaltenes Nanoaggregate Structure: from Perpendicular to Paralleled Orientation at the Water-in-Oil Emulsions Interface. RSC Advances 2017, 7, 3819338203.

(83) Ruiz-Morales, Y.; Mullins, O. C. Coarse-Grained Molecular Simulations to Investigate Asphaltenes at the Oil-Water Interface. Energy Fuels 2015, 29, 1597-1609.

(84) Ervik, Å.; Mejía, A.; Müller, E. A. Bottled SAFT: a Web App Providing SAFT- $\gamma$ Mie force Field Parameters for Thousands of Molecular Fluids. J. Chem. Inf. Model. 2016, 56, 1609-1614. 
(85) Chapman, W. G.; Jackson, G.; Gubbins, K. E. Phase Equilibria of Associating Fluids: Chain Molecules with Multiple Bonding Sites. Mol. Phys. 1988, 65, 1057-1079.

(86) Chapman, W. G.; Gubbins, K. E.; Jackson, G.; Radosz, M. SAFT: Equation-of-State Solution Model for Associating Fluids. Fluid Phase Equilib. 1989, 52, 31-38.

(87) Chapman, W. G.; Gubbins, K. E.; Jackson, G.; Radosz, M. New Reference Equation of State for Associating Liquids. Ind. Eng. Chem. Res. 1990, 29, 1709-1721.

(88) (a) Wertheim, M. Fluids with Highly Directional Attractive Forces. I. Statistical Thermodynamics. J. Stat. Phys. 1984, 35, 19-34; (b) Wertheim, M. Fluids with Highly Directional Attractive Forces. II. Thermodynamic Perturbation Theory and Integral Equations. J. Stat. Phys. 1984, 35, 35-47; (c) Wertheim, M. Fluids with Highly Directional Attractive Forces. III. Multiple Attraction Sites. J. Stat. Phys. 1986, 42, 459-476; (d) Wertheim, M. Fluids with Highly Directional Attractive Forces. IV. Equilibrium Polymerization. J. Stat. Phys. 1986, 42, 477-492.

(89) Müller, E. A.; Gubbins, K. E. Molecular-Based Equations of State for Associating Fluids: A Review of SAFT and Related Approaches. Ind. Eng. Chem. Res. 2001, 40, $2193-2211$.

(90) Economou, I. G. Statistical Associating Fluid Theory: A Successful Model for the Calculation of Thermodynamic and Phase Equilibrium Properties of Complex Fluid Mixtures. Ind. Eng. Chem. Res. 2002, 41, 953-962.

(91) McCabe, C.; Galindo, A. In Applied Thermodynamics of Fluids; Goodwin, A. R., Sengers, J. V., Eds.; Royal Society of Chemistry: London, 2010.

(92) Kontogeorgis, G. M.; Folas, G. K. Thermodynamic Models for Industrial Applications: from Classical and Advanced Mixing Rules to Association Theories; Wiley: Chichester, UK, 2010. 
(93) Lobanova, O.; Mejía, A.; Jackson, G.; Müller, E. A. SAFT- $\gamma$ Force Field for the Simulation of Molecular Fluids 6: Binary and Ternary Mixtures Comprising Water, Carbon Dioxide, and n-Alkanes. J. Chem. Thermodyn. 2016, 93, 320-336.

(94) Herdes, C.; Totton, T. S.; Müller, E. A. Coarse Grained Force Field for the Molecular Simulation of Natural Gases and Condensates. Fluid Phase Equilib. 2015, 406, 91-100.

(95) Steele, W. A. The Physical Interaction of Gases with Crystalline Solids: I. Gas-Solid Energies and Properties of Isolated Adsorbed Atoms. Surf. Sci. 1973, 36, 317-352.

(96) Lemmon, E. W.; McLinden, M. O.; Friend, D. G. In NIST Chemistry WebBook, NIST Standar Reference Database Number 69; Linstrom, P. J., G., M. W., Eds.; Available at: http://webbook.nist.gov (retrieved Aug, 2018).

(97) Ilten, D. F. DETHERM: Thermophysical Property Data for the Optimization of HeatTransfer Equipment. J. Chem. Inf. Comput. Sci. 1991, 31, 160-167.

(98) Lafitte, T.; Avendaño, C.; Papaioannou, V.; Galindo, A.; Adjiman, C. S.; Jackson, G.; Müller, E. A. SAFT- $\gamma$ Force Field for the Simulation of Molecular Fluids: 3. CoarseGrained Models of Benzene and Hetero-Group Models of n-Decylbenzene. Mol. Phys. 2012, 110, 1189-1203.

(99) Jiménez-Serratos, G.; Herdes, C.; Haslam, A. J.; Jackson, G.; Müller, E. A. Group Contribution Coarse-Grained Molecular Simulations of Polystyrene Melts and Polystyrene Solutions in Alkanes Using the SAFT- $\gamma$ Force Field. Macromolecules 2017, 50, 4840-4853.

(100) Van Der Spoel, D.; Lindahl, E.; Hess, B.; Groenhof, G.; Mark, A. E.; Berendsen, H. J. GROMACS: Fast, Flexible, and Free. J. Comput. Chem. 2005, 26, 1701-1718.

(101) Nosé, S. A Molecular Dynamics Method for Simulations in the Canonical Ensemble. Mol. Phys. 1984, 52, 255-268. 
(102) Hoover, W. G. Canonical Dynamics: Equilibrium Phase-Space Distributions. Phys. Rev. A 1985, 31, 1695.

(103) Parrinello, M.; Rahman, A. Polymorphic Transitions in Single Crystals: A New Molecular Dynamics Method. J. Appl. Phys. 1981, 52, 7182-7190.

(104) Nosé, S.; Klein, M. Constant Pressure Molecular Dynamics for Molecular Systems. Mol. Phys. 1983, 50, 1055-1076.

(105) Humphrey, W.; Dalke, A.; Schulten, K. VMD: Visual Molecular Dynamics. J. Mol. Graphics 1996, 14, 33-38.

(106) Stone, J. E. An Efficient Library for Parallel Ray Tracing and Animation. M.Sc. thesis, Univeristy of Missouri-Rolla, 1998.

(107) Arkı, H.; Janke, W. Gyration Tensor Based Analysis of the Shapes of Polymer Chains in an Attractive Spherical Cage. J. Chem. Phys. 2013, 138, 054904.

(108) Martínez-Veracoechea, F.; Müller, E. A. Temperature-Quench Molecular Dynamics Simulations for Fluid Phase Equilibria. Mol. Simul. 2005, 31, 33-43.

(109) Fenistein, D.; Barré, L. Experimental Measurement of the Mass Distribution of Petroleum Asphaltene Aggregates Using Ultracentrifugation and Small-Angle X-Ray Scattering. Fuel 2001, 80, 283-287. 
Graphical TOC Entry

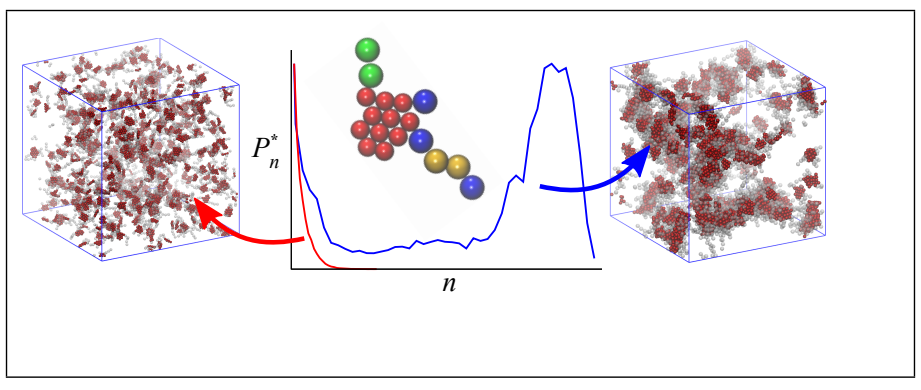

